\title{
OsLG3 contributing to rice grain length and yield was mined by Ho-LAMap
}

Jianping $\mathrm{Yu}^{1 \dagger}$, Haiyan Xiong ${ }^{1 \dagger}$, Xiaoyang $Z \mathrm{Zu}^{1 \dagger}{ }^{\dagger}$, Hongliang Zhang ${ }^{1}$, Huihui $\mathrm{Li}^{2}$, Jinli Miao ${ }^{1}$, Wensheng Wang ${ }^{2}$, Zuoshun Tang ${ }^{3}$, Zhanying Zhang ${ }^{1}$, Guoxin Yao', Qiang Zhang ${ }^{1}$, Yinghua Pan ${ }^{1,4}$, Xin Wang ${ }^{1}$, M. A. R. Rashid ${ }^{1}$, Jinjie $\mathrm{Li}^{1}$, Yongming $\mathrm{GaO}^{2}$, Zhikang $\mathrm{Li}^{2}$, Weicai Yang ${ }^{3}$, Xiangdong $\mathrm{Fu}^{3}$ and Zichao $\mathrm{Li}^{1^{*}}$ (D

\begin{abstract}
Background: Most agronomic traits in rice are complex and polygenic. The identification of quantitative trait loci (QTL) for grain length is an important objective of rice genetic research and breeding programs.

Results: Herein, we identified 99 QTL for grain length by GWAS based on approximately 10 million single nucleotide polymorphisms from 504 cultivated rice accessions (Oryza sativa L.), 13 of which were validated by four linkage populations and 92 were new loci for grain length. We scanned the Ho (observed heterozygosity per locus) index of coupled-parents of crosses mapping the same QTL, based on linkage and association mapping, and identified two new genes for grain length. We named this approach as Ho-LAMap. A simulation study of six known genes showed that Ho-LAMap could mine genes rapidly across a wide range of experimental variables using deep-sequencing data. We used Ho-LAMap to clone a new gene, OsLG3, as a positive regulator of grain length, which could improve rice yield without influencing grain quality. Sequencing of the promoter region in 283 rice accessions from a wide geographic range identified four haplotypes that seem to be associated with grain length. Further analysis showed that OsLG3 alleles in the indica and japonica evolved independently from distinct ancestors and low nucleotide diversity of OsLG3 in indica indicated artificial selection. Phylogenetic analysis showed that OsLG3 might have much potential value for improvement of grain length in japonica breeding.
\end{abstract}

Conclusions: The results demonstrated that Ho-LAMap is a potential approach for gene discovery and OsLG3 is a promising gene to be utilized in genomic assisted breeding for rice cultivar improvement.

Keywords: GWAS, Linkage mapping, Observed heterozygosity, Ho-LAMap, OsLG3, Grain length, Rice domestication, Artificial selection, Genetic interaction

\section{Background}

Rice (O. sativa L.) is a staple food and the world's most important cereal crop. Grain yield is determined by three component traits, namely grain weight, number of grains per panicle, and number of panicles per plant. Grain size is a prime breeding target, as it affects both yield and quality. Therefore, the study of grain size is highly important for the improvement of rice yield and quality as well as for the understanding of the rice domestication process [1]. Grain size is specified by its

\footnotetext{
* Correspondence: lizichao@cau.edu.cn

${ }^{\dagger}$ Equal contributors

'Key Laboratory of Crop Heterosis and Utilization, Ministry of Education/ Beijing Key Laboratory of Crop Genetic Improvement, China Agricultural University, Beijing 100193, China

Full list of author information is available at the end of the article
}

three dimensions (length, width, and thickness). Recently, although a number of quantitative trait loci (QTL) conferring grain length [2] have been isolated, and among them several QTL, such as GS3, GW2, GL3.1, TGW6, qSW5, and GW8, have been well studied [1,3-7], molecular characterization of these and many more unknown genes is still largely unclear. Thus, it is of great significance to understand the underlying genetic and molecular bases of grain length [3].

Recent studies isolated and characterized genes involved in QTL using map-based cloning techniques based on linkage mapping [2, 6, 8-11]. However, for fine mapping, very large sample sizes are required; typically, thousands of individuals are needed and the fieldwork involved is extremely laborious, usually involving 
measurement of multiple traits at several time points across diverse environments. Genome-wide association analysis (GWAS) is generally considered an effective tool to infer causative links between genomic markers and phenotype in many crops [12-16]. However, in previous studies, the causal polymorphisms showed a weaker association than the peak single nucleotide polymorphism (SNP) in Arabidopsis thaliana and rice [12, 17]. Extensive computer simulations have shown that the power of GWAS is low for polygenic traits and that spurious associations can be expected [18]. Linkage mapping was shown to be a valuable complementary approach to address these situations in maize and Arabidopsis [14, 16, 19]. Recently, there have been attempts to combine the vigor of linkage mapping with GWAS in rice [20-22]. GWAS should be performed in conjunction with genetic linkage analysis to detect relevant loci [17].

It is generally agreed that GWAS in rice cannot resolve a single gene due to the low rate of linkage disequilibrium decay. With the development of next generation sequencing technology, DNA sequencing has become easier and cheaper. A core collection of 3000 rice accessions from 89 countries were deep resequenced $[23,24]$, providing an unprecedented resource for genomic research. Herein, we propose a unified approach for gene discovery in rice from candidate region association mapping combing linkage mapping that limits the major problem caused by false positives. We not only describe a new strategy to identify previously unknown but agronomically important alleles based on deepsequencing technology, but also report on the cloning and characterization of a dominant QTL, OsLG3, as a positive regulator of grain length. Natural variations in the OsLG3 promoter region confer grain length and weight and its favorable allele represents a valuable genetic resource for rice cultivar improvement.

\section{Results}

Ninety-nine QTL for grain length were detected by GWAS based on high-density SNPs

We investigated grain length in a diverse panel of $504 \mathrm{cul}-$ tivated rice varieties from worldwide sources (Additional file 1: Figure S1 and Additional file 32: Table S1, Additional file 33: Table S2) grown in five environments at two different latitudes (Additional file 33: Table S3). The panel consisted of the mini core collection [25] and varieties in the International Rice Molecular Breeding Network [26] and have, as part of the rice 3000 genome project, been sequenced with an average sequencing depth of $14.9 \times$ (Data access). After aligning the reads against the rice reference genome of the temperate japonica variety, Nipponbare, we identified a total of 10 million SNPs (1 SNP per $40 \mathrm{bp}$ on average) from these accessions and $3,585,229$ SNPs with missing rates of less than $30 \%$ and a
0.05 minor allele frequency (MAF). GWAS was performed on different populations using the naïve model (LM), general linear model (GLM) with population structure ( $\mathrm{Q}$ matrix), and compressed mixed linear model (CMLM) by controlling population structure and kinship, denoted by the $\mathrm{Q}+\mathrm{K}$ model according to $\mathrm{Yu}$ et al. [27, 28] (Additional file 3: Figure S3a-c, Additional file 34: Table S5, Additional file 35: Table S6, Additional file 36: Table S7, Additional file 37: Table S8, and Methods). LM not only detected all the cloned genes and published QTL related to rice grain length, but also indicated a whole genome association signal in all types of populations, and thus included a very high number of false positive associations (Additional file 3: Figure S3a). When using CMLM and setting $-\log _{10}(P)=7.9(P=0.05 / \mathrm{n}, \mathrm{n}$ denotes the number of all SNPs), we detected only two, two, and four QTL in the full, indica, and japonica populations, respectively, of which one is the cloned gene GS3, one is a published QTL in both full and indica populations, and one is a published QTL in japonica (Additional file 3: Figure S3b and Additional file 34: Table S5). When using GLM and the same $-\log _{10}(P)$, we detected 65,30 , and 53 QTL in the full, indica, and japonica populations, respectively, and these QTL hit seven (GW2, GS3, GW5, GW6a, TGW6, GL7, and GLW7), two (GS3 and GW5), and three (GW2, GL7, and GLW7) cloned genes, respectively, and about $65 \%, 53 \%$, and $57 \%$ of the published QTL (Fig. 1a, b and Additional file 35: Table S6, Additional file 36: Table S7, Additional file 37: Table S8). However, four genes from map-based cloning (GS2, GL3.1, GS5, and GW8) were not identified by the model because of the rarity of causal variants, or strong correlation between causal variation and population structure. Most of the genes being separated by map-based cloning involve rare causal variations and cannot be identified by GWAS, such as GS2 and GL3.1 (Additional file 33: Table S9). Some other genes cannot be detected by GLM because the frequency of their causal variation confound with population structure (Additional file 4: Figure S4). For example, even though GW8 and GS5 displayed the high association signals in LM $\left(-\log _{10}(P)=\right.$ 19.0 and 17.8), their signal in GLM decreased to 1.2 and 4.9, and GW8 indicated no significance by simple t-test when removing effect of $\mathrm{Q}$ structure from their original phenotypes (Additional file 5: Figure S5). Taken together, these results suggested that there were still many valuable genes for grain length that remained to be cloned.

\section{Co-localized QTL for grain length by joining GWAS and multiple biparental linkage analysis}

We used four segregating populations from cross involving six varieties to identify QTL for grain size and grain weight, including SLG-1 (SLG, with the largest grain), Chuanqi (CQ, with the smallest grain), Nipponbare (NIP, medium grain), Haobuka (HBK, large grain), IRAT109 


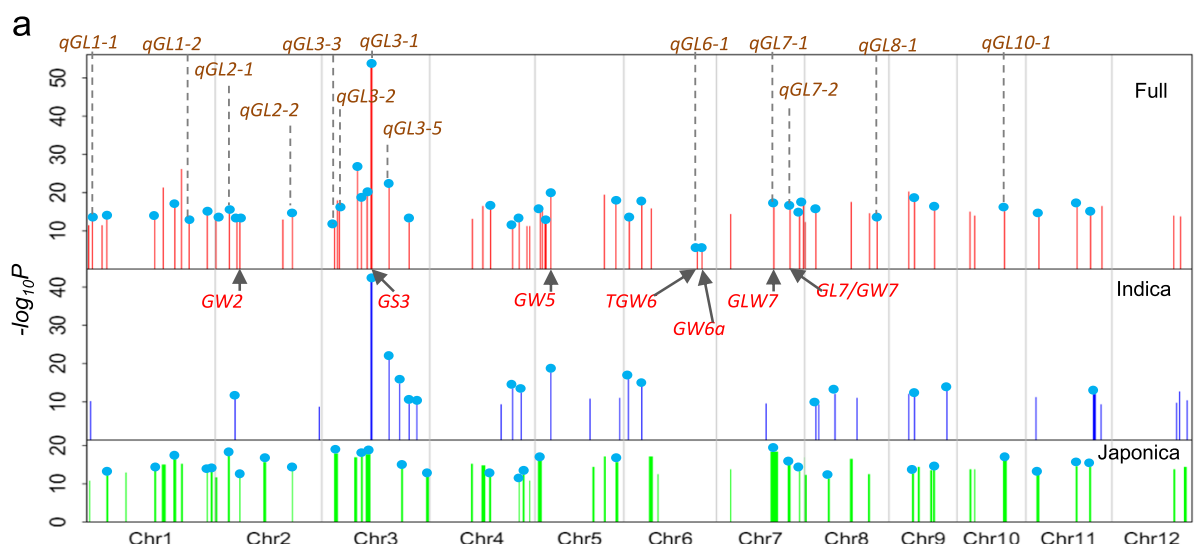

b
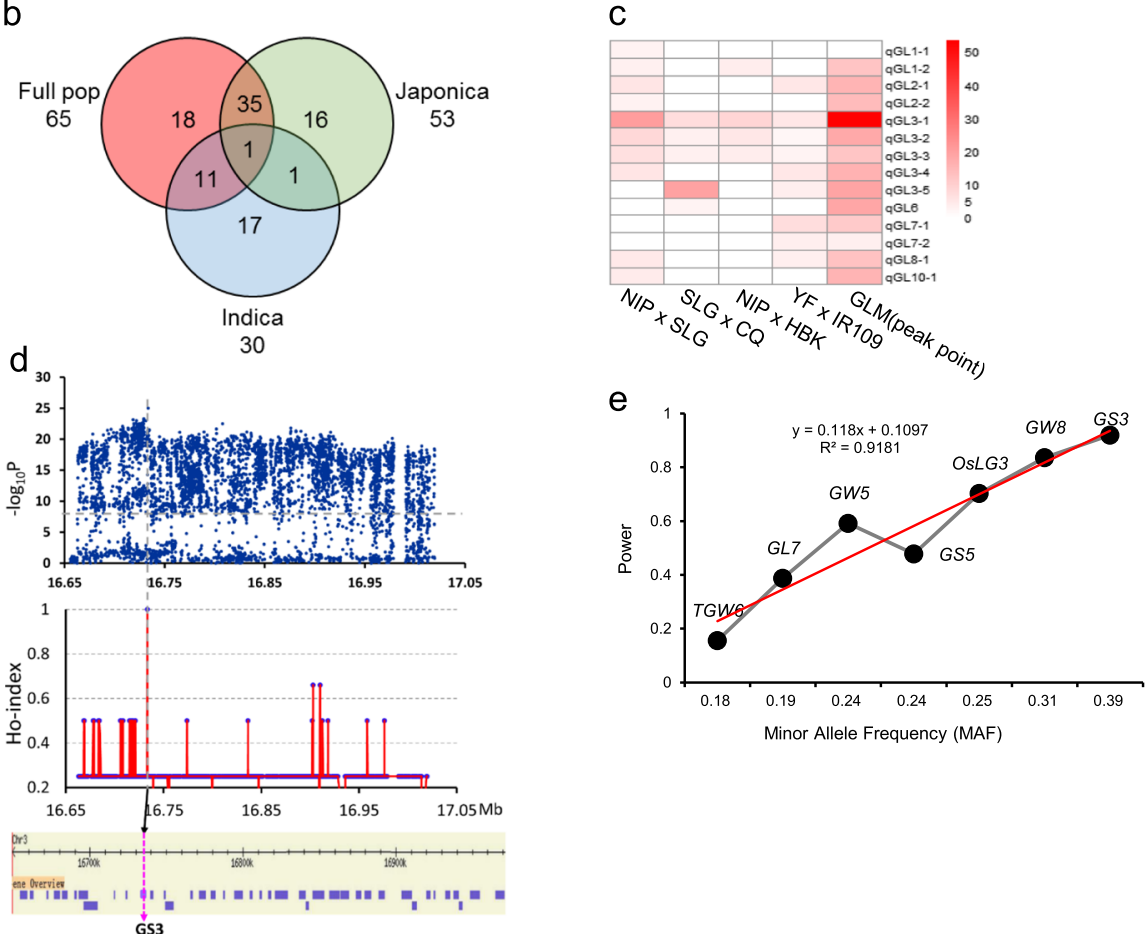

Minor Allele Frequency (MAF)

Fig. 1 Ho-LAMap accelerated the identification of grain size loci in rice. a Comparison of GWAS results for grain length among the full, indica, and japonica populations using the GLM $(\mathrm{Q})$ model. Arrows indicate the physical positions of known grain length genes overlapping with the QTL. Vertical dashed lines represent QTL co-localized with linkage mapping. Blue dots indicate QTL overlapping with the published QTL. b Venn diagram showing numbers of unique and shared QTL mapped by GWAS among the full, indica, and japonica populations. c Heat map for effect of grain length QTL mapped by four linkage populations and GWAS. Rows in the heat map correspond to the 14 QTL for grain length. $\mathbf{d}$ Identification of causal SNPs in QTL qGL3-1 using Ho-LAMap, a novel method, which combined linkage mapping and association mapping for grain length. Manhattan plot for candidate region association mapping for qGL3-1 (top) and Ho index plots of qGL3-1 (middle). Red regression lines were obtained by averaging SNP indices from a moving window of two consecutive SNPs, and shifting the window one SNP at a time. The bottom part corresponds to annotated genes in this region, with the purple arrow representing GS3. e Minor allele frequency influence on effectiveness of Ho-LAMap

(large grain), and Yuefu (YF, medium grain), which were selected from the MCC1 panel and showed high genetic differentiation from each other (Additional file 7: Figure S7 and Additional file 33: Table S10). The four segregating populations included BILs (backcross inbred lines) from Nipponbare and SLG-1, CSSLs (chromosome segregation substitution lines) from YF and IRAT109, $\mathrm{BC}_{1} \mathrm{~F}_{2}$ lines from Chuanqi and SLG-1, and $\mathrm{BC}_{1} \mathrm{~F}_{2}$ lines from
Nipponbare and Haobuka. Substantial variation in grain size traits was observed among these populations (for example, 07DH010-14, Additional file 8: Figure S8). Thirty-four QTL for grain shape and weight were detected among the four groups (Additional file 9: Figure S9 and Additional file 33: Table S11). Among them, 13 QTL for grain length co-localized with QTL mapped by our GWAS (Fig. 1a, c). Of 34 QTL, 22 were detected in at 
least two combinations. Moreover, three QTL (qGL3-1, $q G L 3-2$, and qGL3-3) for grain length were detected in four crosses (Fig. 1c).

\section{The efficiency of Ho-LAMap for detection of causal genes and simulation studies}

Similar to other reports of GWAS in rice [12, 13, 29], the peak SNPs were not part of the causal genes except for GS3, even though we narrowed the QTL regions of several hundred kilobases (Additional file 6: Figure S6). However, analysis of the causal alleles of some cloned genes indicated that most of the varieties shared the same causal variations [5-7], although the varieties did not uniformly share the same non-causal variations. Based on this, we propose a strategy - Ho-LAMap that joins GWAS and multiple biparental linkage analysis to distinguish the causal gene from other genes confounded with population structure. The principles of Ho-LAMap are depicted in Additional file 12: Figure S12 and Additional file 33: Notes S3. Firstly, we cross diverse founder varieties (i.e., varieties that are significantly different from reference parent for grain traits) with a reference parent (usually a small grain variety). After backcrossing or several self-pollinated generations, advanced populations are prepared for QTL mapping and these serve to identify the regions in the genome that are most likely to carry the causal genes. Primary fine mapping will help to ensure the boundary of the QTL. Association mapping using the GLM (Q) model in the QTL interval will identify almost all SNPs that correlate significantly with the target trait. In crosses that have detected the targeted QTL, the majority of SNPs within the QTL interval will segregate in a 1:1 founder varieties:reference parent ratio. However, the SNP responsible for the change of phenotype will be the same in all founder parents detecting the targeted QTL. If we define the Ho (observed heterozygosity per locus) index as the ratio between the number of heterozygous crosses corresponding to each SNP locus and the total number of crosses which have the targeted QTL, we expect this index to equal 1 near the causal SNP and 0.5 for the unlinked loci. Ho indices can be scanned across the genome to find the region with a Ho index of 1 , and presumably harboring the gene responsible for the change of phenotype. In this method, we just need to sequence several parents and do SNP mapping. Our proposed name for this approach is Ho index unified linkage and association mapping (Ho-LAMap) as applied to rice.

Among three co-localized QTL, qGL3-1 encompassed the cloned gene GS3 [3] and thus was used to validate the efficiency of Ho-LAMap. In the mapping region of qGL3-1 overlapping between GWAS and multiple biparental linkage analysis, we detected 1763 SNPs with significant association signals. For each identified locus, we obtained the Ho index between each pair of parents for the four combinations, and plotted the Ho indices in the overlapping mapped region (Fig. 1d). The Ho index of GS3 was 1 and those for almost all other loci were below 0.5 , and even 0 . The results confirmed that this approach allowed us to rapidly identify the causal gene according to GWAS combined with primarily mapped QTL common among multiple biparental crosses.

Given the success Ho-LAMap in identifying genes controlling quantitative traits based on common QTL and GWAS, we are more generally interested in the possible factors affecting the efficiency of Ho-LAMap in detecting causal genes. Simulation studies (see Methods) on several known genes, such as GS3, TGW6, etc., were carried out to estimate (1) the number of crosses required $(\mathrm{N}),(2)$ the minor allele frequency of the targeted gene, and (3) the subgroup that selected parents were from. Our results suggest that the minor allele frequency is perhaps the most important factor for effectiveness of Ho-LAMap and that parents selected from an appropriate subgroup would increase the efficiency (Fig. 1e and Additional file 13: Figure S13). Whereas the resolving power increased with the number of crosses we concluded that, in application of Ho-LAMap to complex quantitative traits in rice, $\mathrm{N} \geq 4$ should be required for genes such as TGW6 (Additional file 14: Figure S14 and Additional file 33: Table S12).

\section{Identification of two new grain length loci via Ho-LAMap} We further applied Ho-LAMap to qGL3-2 and qGL3-3 and successfully identified two new genes for grain length (Fig. 2a, Additional file 15: Figure S15 and Additional file 33: Notes S1, S2). Herein, we focused on qGL3-3 and identified a small genomic region harboring a cluster of SNPs with a Ho index of 1 (Fig. 2a): qGL3-3 showed a cluster of five SNPs with a Ho index of 1 . Then, we examined the SNPs with Ho indices of 1 in detail. All five SNPs were localized to the promoter of gene Os03g0183000 (OsLG3) (Additional file 33: Table S13), which encodes an APETALA2/ethylene-responsive element binding protein 125 . We compared genomic sequences corresponding to the ORF and the promoter regions of OsLG3 between varieties with short and long grain and found that the promoter region had high nucleotide diversity (Additional file 16: Figure S16). Significantly, three varieties with long grains (IRAT109, SLG-1, and Haobuka) had the same promoter sequences that were different from those of all short-grain varieties (Additional file 33: Table S14). We assayed the temporal and spatial expression patterns of OsLG3 in NIL(SLG) and NIL(NIP) using quantitative real-time PCR (qRT-PCR) with total RNA from 13 tissues. The transcript was much more abundant in NIL(SLG) than in NIL(NIP) in young panicles and developing rice endosperms (Fig. $2 \mathrm{~b}$ and Additional file 17: Figure S17). Such expression differences 


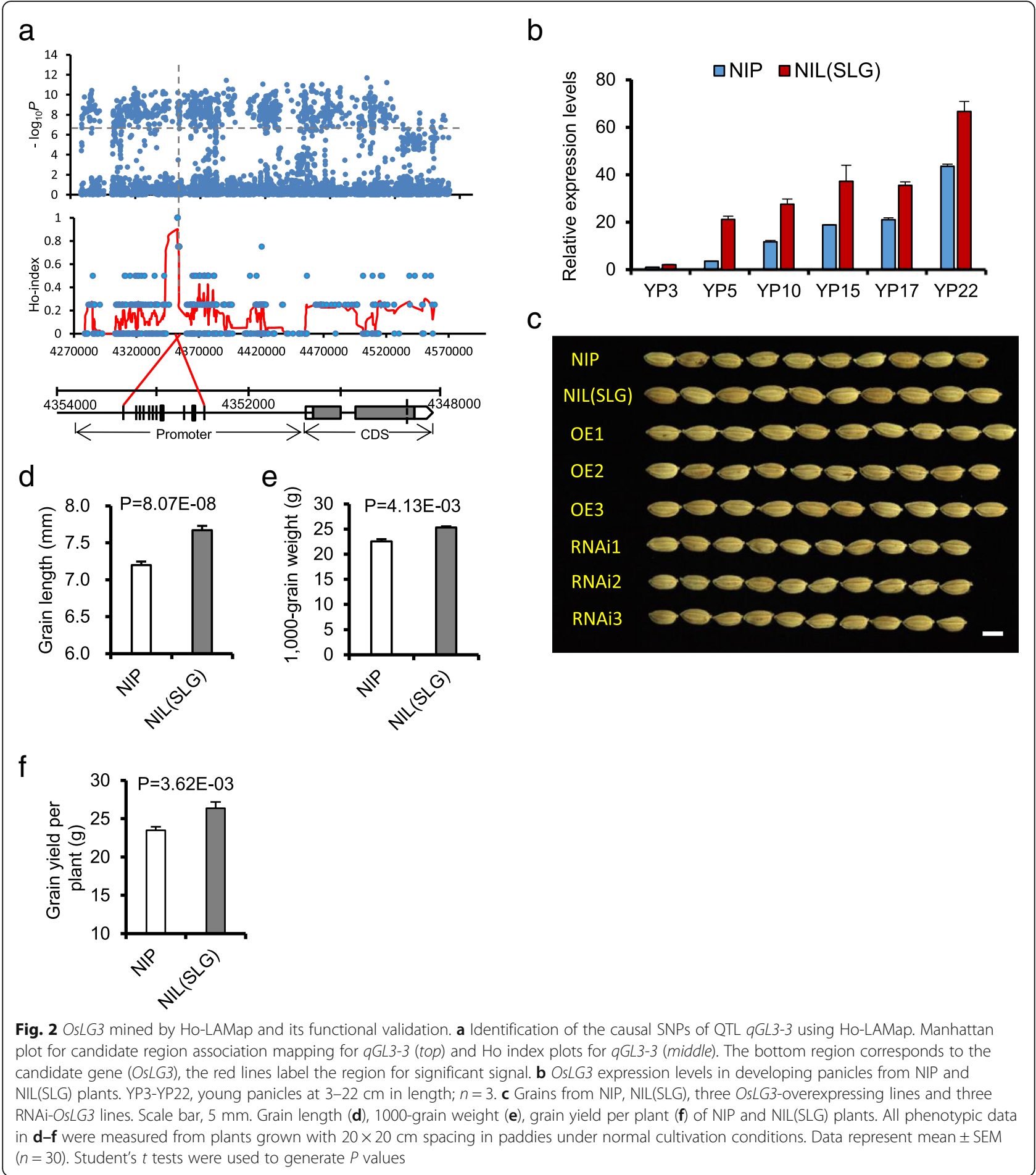

corresponded well with the critical stages for determination of grain length and grain weight [30]. Therefore, we hypothesized that differences in expression levels of OsLG3, attributable to polymorphisms in the promoter rather than the coding variation, were key determinants of variation in grain length.
Two transformation constructs were prepared to test this hypothesis. The first, OE, contained the cDNA of OsLG3 from Nipponbare (short grain) driven by the $35 \mathrm{~S}$ promoter; the second, contained RNAi-OsLG3, the fragment of OsLG3 coming from Nipponbare. All of the transgenic lines that overexpressed the Nipponbare 
OsLG3 allele showed increased grain length and grain weight, compared to transgene-negative plants (Fig. 2c and Additional file 18: Figure S18a-c). All transgenic RNAi-OsLG3 plants formed grains that were substantially shorter and lighter than those from transgene-negative plants (Fig. 2c and Additional file 18: Figure S18d, e). Thus, a longer grain could be caused by the variations in the promoter region.

\section{OsLG3 regulates grain length by altering cell number but} does not influence grain quality

Although most of the morphological characteristics of NIL(SLG) were similar to those of Nipponbare (Additional file 19: Figure S19), the spikelet hulls of NIL(SLG) plants before fertilization were longer than those of Nipponbare plants. We conducted scanning electron microscopy study of outer and inner surfaces of glumes from NIL(SLG) and NIP (Additional file 20: Figure S20). There was little, if any, difference in cell length in either the palea or lemma, but total cell numbers of outer and inner epidermal cells in the longitudinal direction in NIL(SLG) were more than their equivalents in Nipponbare. We also investigated the expression of the key genes determining cell cycle time $[7,31]$ such as CDKA1, CYCD3, MCM3, CYCA2.1, CYCA2.2, CYCA2.3, $C A K 1$, and $H 1$. The transcript levels of most of these genes were considerably higher in NIL(SLG) plants relative to Nipponbare plants (Additional file 21: Figure S21), suggesting that the increase in cell number in NIL(SLG) might result from elevated expression of genes promoting cell proliferation. In addition, compared with Nipponbare, NIL(SLG) had a $4.2 \%$ higher grain length to width ratio, but had the same grain chalkiness level and the same starch granule appearance in transverse sections of grains (Additional file 22: Figure S22). These observations suggest that OsLG3 might promote longitudinal growth by increasing cell proliferation while not influencing grain quality (Fig. 2d-f and Additional file 23: Figure S23 and Additional file 24: Figure S24).

\section{Expression patterns of $O s L G 3$ and its transcription activator activity}

As mentioned above, OsLG3 transcripts were detected in various tissues by qRT-PCR analysis (Fig. 2b and Additional file 17: Figure S17). To determine the spatial expression pattern of OsLG3 in detail, we generated transgenic plants containing OsLG3 promoter::GUS fusions (ProOsLG3::GUS). We observed GUS activity in all of the analyzed tissues/organs (roots, stems, sheaths, leaves and panicles) (Additional file 25: Figure S25). Higher GUS activity was detected in developing panicles, spikelet hulls during spikelet development and roots (Additional file 25: Figure S25). These results indicate that $O s L G 3$ is a temporally and spatially expressed gene.
As OsLG3 encodes an AP2 domain class transcription factor, we speculated that OsLG3 is localized in the nucleus. To determine subcellular localization of OsLG3, we expressed an OsLG3-green fluorescent protein (GFP) fusion protein under the control of the $35 \mathrm{~S}$ promoter in onion epidermal cells. As shown in Fig. 3a, GFP fluorescence in 35S: OsLG3-GFP transgenic epidermal cells was observed exclusively in nuclei. Thus, this result suggests that OsLG3 is a nuclear-localized protein, consistent with its proposed function as a transcription regulator.

We further tested the activity of a set of truncations and deletions of OsLG3/OsAP2-125. It showed that the C-terminal $168-334$ aa region is sufficient to activate the reporter, whereas the N-terminal truncated OsLG3/ OsAP2-125 proteins are not (Fig. $3 \mathrm{~b}$ ). These results show that transcription activation activity of OsLG3/OsAP2-125 resides in its $\mathrm{C}$-terminal regions and that DNA-binding activity resides in its $\mathrm{N}$-terminal regions containing the AP2 domain [32, 33].

\section{Validation of functional variations in promoter region and origin of OsLG3}

We next sequenced the OsLG3 promoter regions and measured grain length in 283 rice accessions, including 247 cultivated, worldwide varieties of $O$. sativa in MCC1, and 36 wild accessions originating from a wide geographic range across Asia (Additional file 32: Table S1). On the basis of the nucleotide polymorphisms identified by Ho-LAMap, we could divide the sequences of the cultivated varieties into four haplotypes that were placed into two classes by phylogenetic analysis: haplotypes 1 and 3 in class A and haplotypes 2 and 4 in class B (Fig. 4a, b). Cultivars having class A haplotypes showed significantly higher OsLG3 expression levels and a longer grain phenotype than those with class B haplotypes by qRT-PCR analysis (Fig. 4c, d). Furthermore, transient assays indicated that the four consensus SNPs, rather than indel1, played a significant role in regulating OsLG3 expression differences between genotypes, resulting in diversity on grain length (Fig. 4a, e).

Geographically, 32 temperate japonica cultivars with Hap4 were from northern China, Russia, Korea, and Japan, or higher elevation zones (Fig. 4f). In contrast, samples with Hap1, including 133 indica and 10 javanica cultivars, were from South China, Southeast Asia, East Africa and South America. This group also included 19 glutinous temperate japonica from the Yunnan-Guizhou Plateau or mixtures. The 26 O. rufipogon samples with Hap1 were from Southeast Asia and south China, whereas the 8 Hap4 O. rufipogon samples originated from Guangxi province in China (Additional file 32: Table S1). These observations indicate that a north-south differentiation in OsLG3 occurred between indica and japonica during rice domestication. 
a
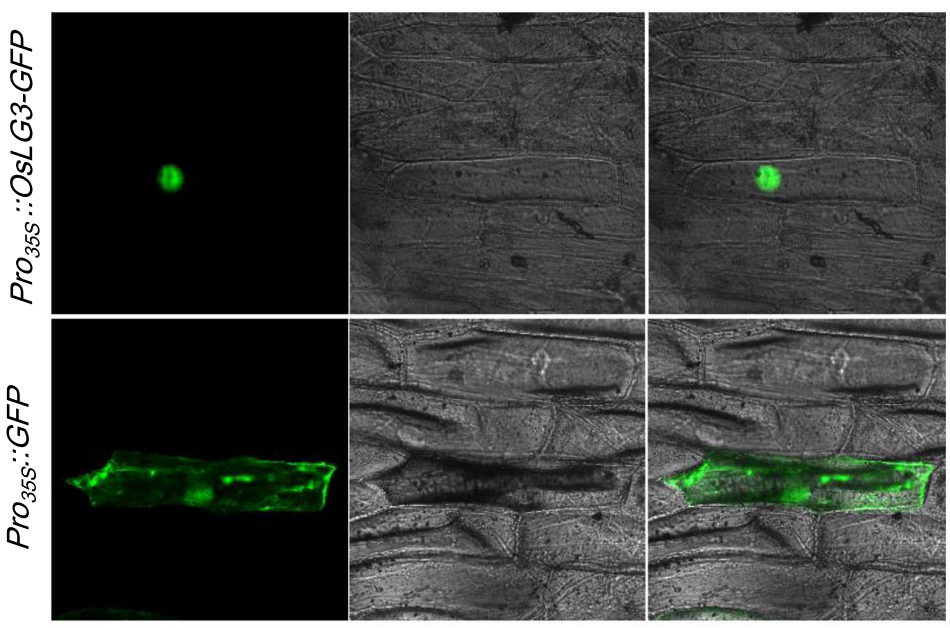

b

GFP

Bright field

Merged

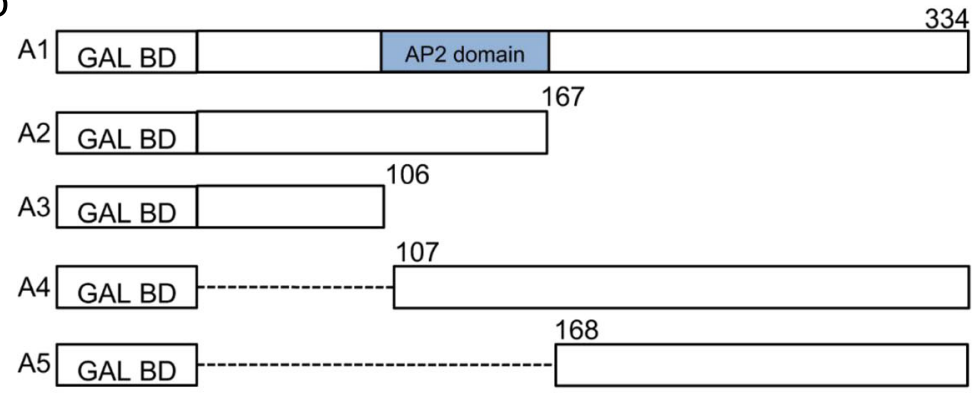

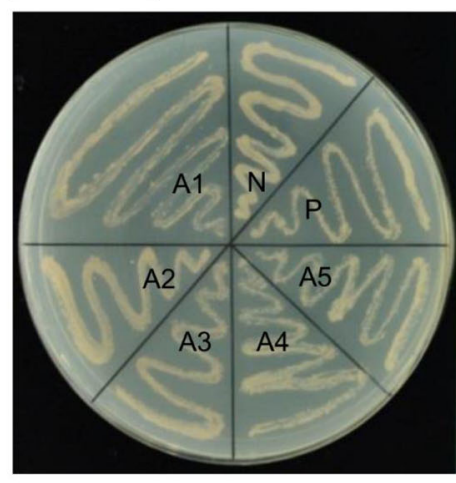

$\mathrm{SD} /$-Trp

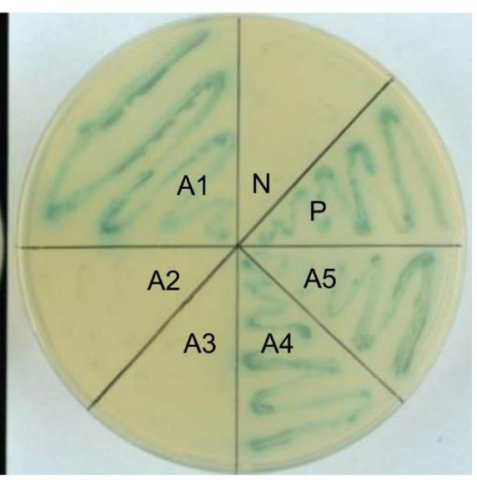

SD/-Trp/-His/-Ade/X-a-gal

Fig. 3 OsLG3 is a nuclear-localized transcriptional activator. a Subcellular localization of OsLG3. b Transactivation assay of truncated OsLG3. Fusion proteins of the GAL4 DNA-binding domain and different portions of OsLG3 were expressed in yeast strain AH109. A1 indicates the full-length coding sequence of OsLG3; A2 to A5 indicate the mutated forms of OsLG3 (nucleotide positions were labeled in the diagrams), respectively. P and $\mathrm{N}$ indicate the positive and negative control, respectively. The culture solution of the transformed yeast was dropped onto the control plate $\mathrm{SD} /$-Trp or selective plate (SD/-Trp/-Ade/-His/X-a-gal). The plates were incubated for 3 days

We analyzed the genetic diversity [34] in OsLG3 and its flanking regions $(\sim 400 \mathrm{~kb})$ to determine whether OsLG3 had undergone artificial selection during domestication of indica. Clearly decreased nucleotide diversity was observed at the OsLG3 locus in indica, but not in temperate or tropical japonica accessions (Fig. 4g). The overall average nucleotide diversity of the $20 \mathrm{~kb}$ OsLG3 flanking regions was higher than that of OsLG3 in indica, but also significantly lower than in wild rice
(Fig. 4h). The diversity in an approximately 100-kb region around OSLG3 in indica was lower than that in japonica and wild rice. These results suggest that the decreased diversity in indica resulted from selection.

To gain deeper insights into the evolution of OsLG3, we analyzed all polymorphic sites in the entire gene region of a larger population. We identified 7 haplotypes in 504 diverse cultivars (3 indica and 4 japonica haplotypes) and 13 haplotypes in 15 wild accessions. A 


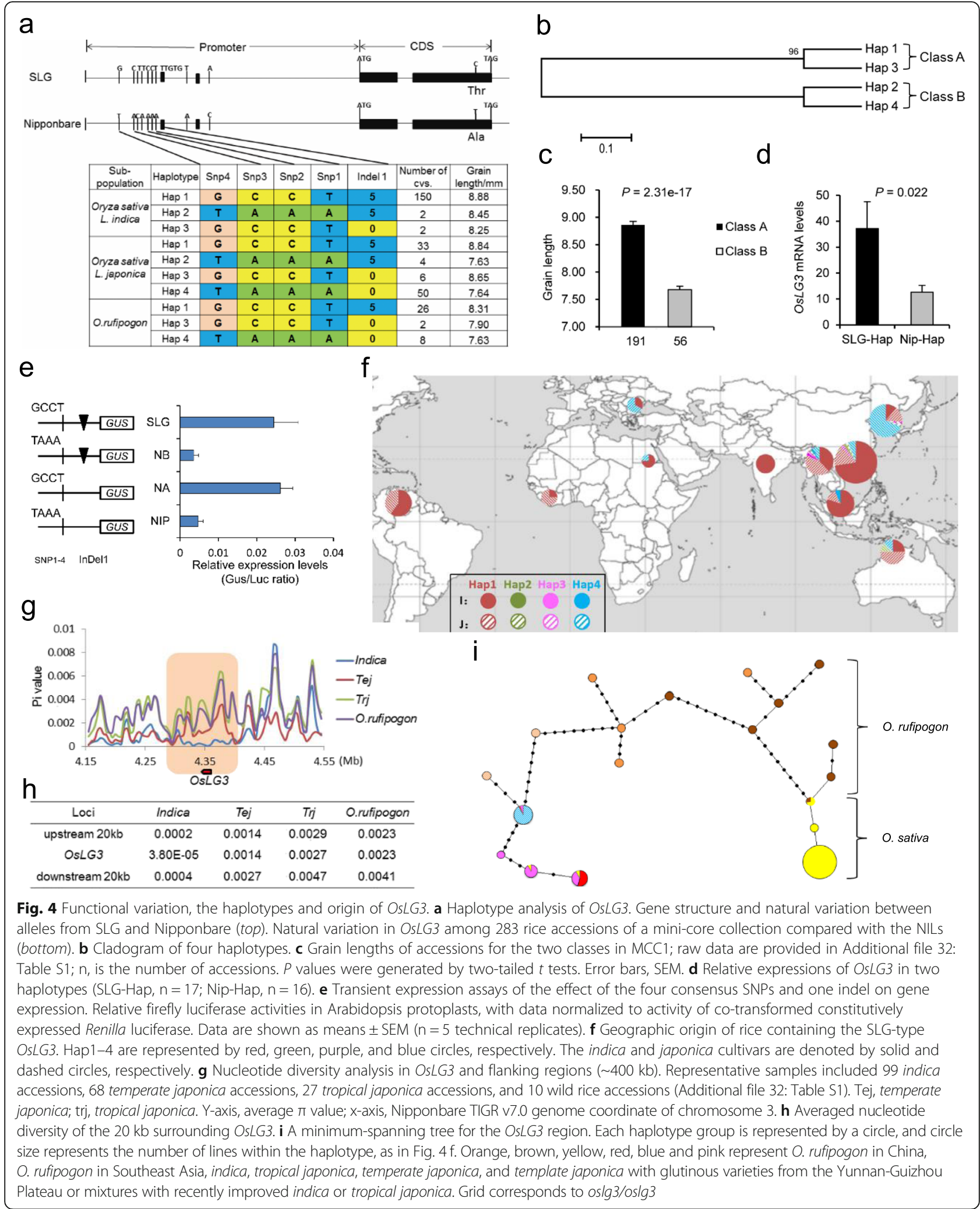

minimum-spanning tree of haplotypes revealed two distinct clusters - separate cultivar and wild rice haplotype clusters - in OsLG3. The cultivars divided into two subgroups, indica and japonica (Fig. 4i). The wild accessions contained both large and small grain haplotypes. Only some $O$. rufipogon accessions from China had 
small grain haplotypes, which might have been inherited to ancient japonica. All indica haplotypes had large grain OsLG3 haplotypes and one haplotype was present in two wild accessions, indicating the large grain haplotypes in indica might be derived from an O. rufipogon accession with a large grain haplotype. The large grain allele of OsLG3 in javanica and some glutinous template japonica from the Yunnan-Guizhou Plateau or admixed accessions may be derived from indica sources (Fig. 4f, i). Our results implied that OsLG3 alleles in indica and japonica independently originated from distinct ancestors, consistent with previous conclusions that the two subspecies were domesticated from distinct ancestral gene pools [35].

\section{Genetic interactions with other related genes and potential utilization in rice breeding improvement}

To dissect the genetic interaction between OsLG3 and other genes controlling grain length, we analyzed the promoter sequences of OsLG3 and various haplotypes of GS3, GW8, TGW6, GW2, and GL3.1 in 498 accessions (Additional file 32: Table S1, Additional file 33: Table S2). By re-sequencing our germplasm resources, we found that the alleles of GW2 and GL3.1 responsible for longer grains were quite rare. We then investigated OsLG3, GS3, GW8, and TGW6 and analyzed the association between their grain length and allelic variants. All varieties were categorized by allelic variations in the functional nucleotide polymorphisms (FNPs) of OsLG3, GS3, GW8, and TGW6. The beneficial allele of OsLG3 had an epistatic effect on grain length for GW8 and TGW6; however, the beneficial allele of GS3 had a recessive epistatic effect on grain length for OsLG3 (Fig. 5a-c and Additional file 26: Figure S26). Our results suggest these loci cooperatively regulate grain length in rice, as accessions with the same haplotype for a given gene exhibited grain length diversity, and that variations at OsLG3 and GS3 are major factors affecting grain length diversity on a wide range of genetic backgrounds (Fig. 6a). Phylogenetic analysis showed that landrace varieties and improved varieties in the indica group were distinguished remarkably on account of improvement of grain length, although it fitted well within the japonica group. This indicated that grain length in indica rice has been widely improved by the presence of beneficial alleles of GS3 and OsLG3, whereas there remains room for improvement for grain length in japonica (Fig. 6a, c and Additional file 27: Figure S27). In the japonica group, grain of some improved varieties was significantly longer because of introgression of the beneficial allele of OsLG3 (Fig. 6a, b). Our results suggest that OsLG3 has potential for improvement of grain length in japonica.

\section{Discussion}

Grain size traits of rice are complex and generally controlled by multiple genes. Genetic control of these characteristics has been investigated over the last decade. To date, a total of 11 QTL for grain size (including GS3, GL3.1, GW6a, TGW6, GL7, GLW7, GS5, GW5, $G W 8, G S 2$, and $G W 2$ ) have been cloned from natural rice varieties by a classic map-based approach. Despite these efforts, the mechanisms that establish the final size of grain remain poorly understood. In this research, we conducted GWAS for rice grain length based on high density SNPs (an average of 1 SNP per $40 \mathrm{bp}$ ) from 504 accessions and identified 99 QTL for grain length. This provides important information for cloning novel grain length genes in the future. Our findings confirmed that Ho-LAMap was able to mine genes rapidly over a wide range of experimental variables using deep-sequence
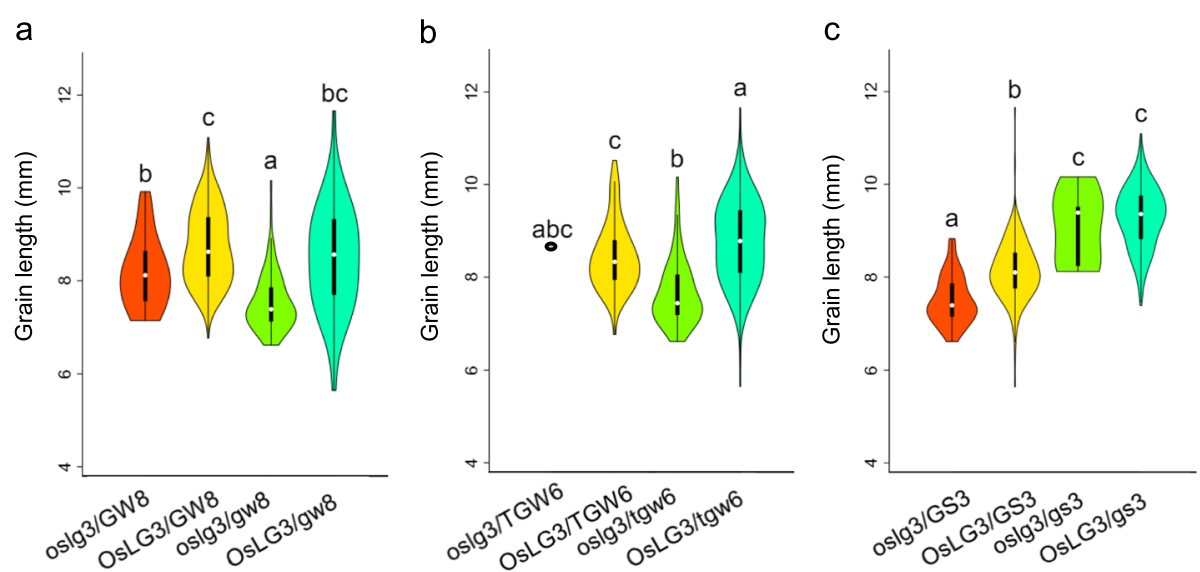

Fig. 5 The genetic interactions between OsLG3 and other grain length-related genes based on diverse germplasm. A box plot and a kernel density plot were generated as violin plots for different groups. a-c Relationship between OsLG3 and other grain size-related genes, including GS3 (a), GW8 (b), and TGW6 (c). The violin map was constructed in R. Different letters above columns indicate statistically significant differences between groups (Tukey's honestly significant difference test, $P<0.05$ ). Landraces, genotypes, and phenotypes are listed in Additional file 32: Table S1 

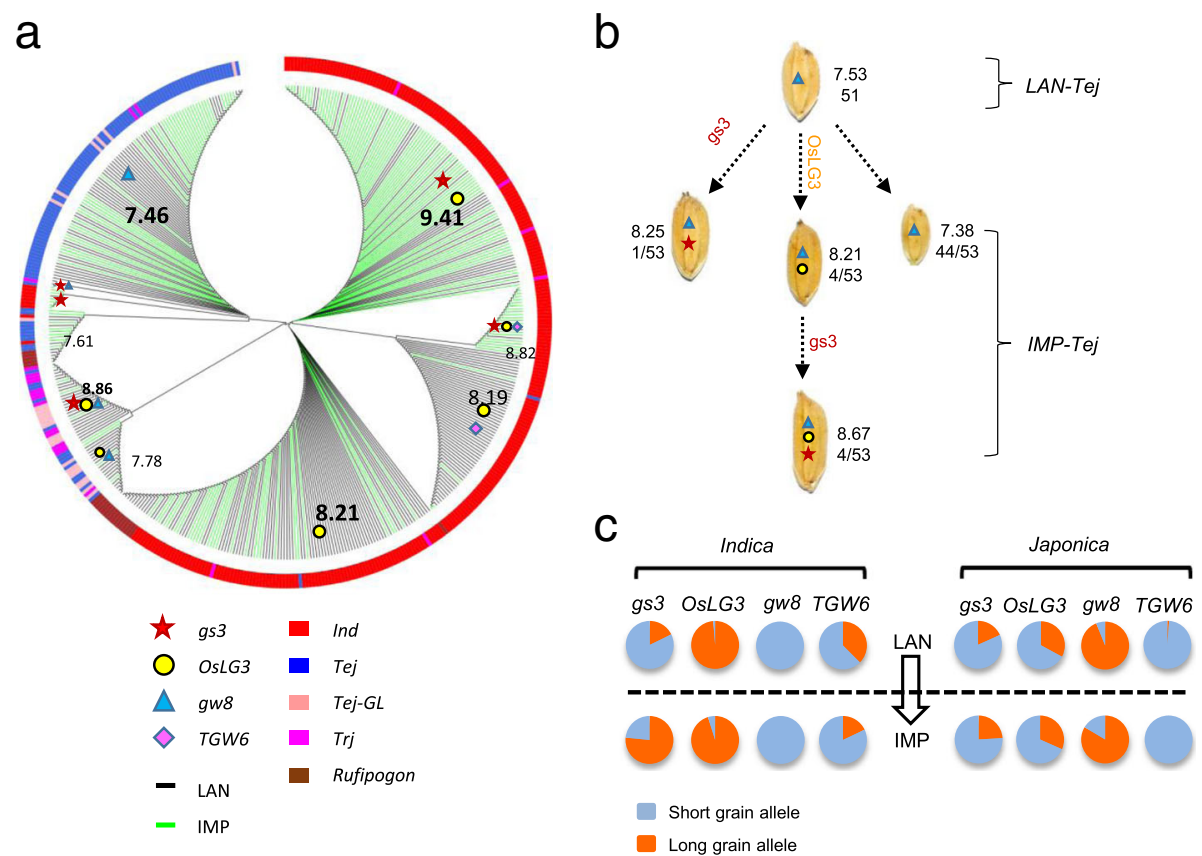

Fig. 6 Breeding improvement of OsLG3. a Phylogenetic analysis of four grain length-related genes in 480 accessions. The phylogenetic tree of 480 varieties was constructed based on different functional SNPs or indels (listed in Additional file 32: Table S1) by MAGE 6.0. All varieties were categorized by allelic variations in the FNP of OsLG3, GS3, GW8, and TGW6. The red pentagram refers to the beneficial allele of gs3, yellow dot to the beneficial allele of OsLG3, blue triangle to the beneficial allele of GW8, purple prism to the beneficial allele of $T G W 6$, black lines to landrace varieties and green lines to improved varieties. Different colors reflect the different subgroups, with its abbreviation as in Fig. 4. b Improvement of haplotype combination for four grain length-related genes, as in $\mathbf{a}$. Top numbers indicate average grain length; bottom numbers correspond to accessions number with a type of haplotype combination in the subgroup. c Spectra of allele frequencies comparing landrace and improved varieties at the causal polymorphisms of GS3, OsLG3, GW8, and TGW6 during modern breeding in the respective subgroups. LAN landraces, IMP improved varieties

data and that natural variations in the OsLG3 promoter region conferred grain length and weight.

In rice, GS3, GL3.1, GW2, GW5, and GW6a controlled grain size by regulating cell proliferation, whereas GLW7 did so by regulating cell expansion. GS2, GS5, GL7/ GW7, and GW8 influenced both cell division and cell expansion [36]. In this study, we reported a novel transcriptional regulatory factor that controlled grain length by influencing cell proliferation. Expression analysis showed that elevated expression levels of OsLG3 increased the expression levels of many key genes involved in the cell cycle. The glumes from NIL(SLG) had the same cell length with NIP, but cell numbers of epidermal cells in the longitudinal direction in NIL(SLG) were more than those in Nipponbare. Thus, these findings indicate that OsLG3 contributes to grain length by promoting cell division.

Many QTL related to rice grain size or weight were reported to enhance the grain yield. Among them, GW2, GS2, and GW5 increased grain size and yield to a large extent; however, they caused a sudden increase in the percentage and degree of chalkiness, resulting in a sharp decrease in grain quality. Our results revealed that the introduction of SLG allele of OsLG3 into existing elite japonica varieties might increase the grain length, weight and yield without disturbing the grain quality. According to the pedigree records, Yunguang8 is one of the first two-line hybrid rice most widely grown in Yunnan province of China because of its particularly high yield and good appearance quality. Its parents are the Yunhuil1 and Nongken58S, a formerly widely used good-quality PTGMS line in China. A resequencing study showed that Yunguang8 carried the beneficial allele of OsLG3 deriving from Yunhuil1 and the $g w 8^{\text {Basmati }}$ allele deriving from Nongken58S, respectively (Additional file 32: Table S1). Thus, the combination of the $O s L G 3^{S L G}$ and $g w 8^{\text {Basmati }}$ alleles provides a good example, which could have been employed by breeders, for simultaneously improving rice yield and grain quality over what is currently achievable.

Rice is a major cereal and a model system for plant biology, however, the origin of cultivated rice and its domestication history have long been debated. During the past decades, several models were proposed for rice domestication. Asian cultivated rice contains five genetically distinct ecotype groups, namely indica, aus, temperate japonica, tropical japonica, and aromatic. A simple single origin of cultivated rice suggested that the 
cultivated rice was primarily domesticated from the annual genotypes, and the two subspecies indica and japonica were regarded to be molded after domestication [37, 38]. Londo et al. [39] reported that indica and japonica were domesticated independently. Another hypothesis indicated that japonica rice was first domesticated and indica rice was subsequently developed from crosses between japonica rice and another clade of $O$. rufipogon [40]. Recently, Civán et al. [41] suggested that there were three geographically separate domestications of Asian rice and concluded that rice domestication was a multiregional process separately producing the indica, japonica, and aus types of rice. Some well-characterized domestication genes (Sh4, Prog1, Bh4, and qSH1) in rice were found to be fixed in both subspecies with the same alleles, thus supporting the hypothesis of a single domestication origin [42-45]. However, our findings revealed that OsLG3 alleles in indica and japonica independently originated from distinct ancestors (Fig. 4i). In view of the fact that grain size is a target trait for both domestication and artificial breeding, this conclusion was consistent with the hypothesis that indica and japonica were domesticated independently. Although rice is a self-crossing species, in this study, gene flow was detected from domesticated to two (out of the total 15) wild accessions (Fig. 4i). This is in agreement with a previous report of gene flow from domesticated to wild crop in another self-crossing species [46].

As demonstrated by our study, grain length is a complex trait associated with population structure in rice (Additional file 4: Figure S4a and Additional file 5: Figure S5). Although we detected two major QTL using the CMLM model (Additional file 34: Table S5), a large amount of middle effect and minor effect QTL, even many other major QTL for grain length (e.g., GW2, OsLG3, TGW6, $g w 8$ ), could not be identified (Additional file 3: Figure S3). This indicates that not all associations that are eliminated in the CMLM model are false. GWAS incorporating $\mathrm{Q}$ and $\mathrm{K}$ in a CMLM controls $P$ value inflation well, but leads to false negatives, missing some potentially important true associations. It is possibly due to the confounding between the covariates and test marker weakening the signals of Quantitative Trait Nucleotides (QTNs), resulting in false negatives in CMLM [47]. Recently, Liu et al. [47] proposed a new method, FarmCPU (Fixed and random model Circulating Probability Unification), to control false positives as well as CMLM with reduction in false negatives. FarmCPU iteratively performs marker tests with pseudo QTNs as covariates in a fixed effect model and optimization on pseudo QTNs in a random effect model. To some extent, this process removes the confounding between testing markers and kinship, prevents model over-fitting, and controls false positives simultaneously. We reanalyzed our data using
FarmCPU. Seven, four, and two QTL were identified by FarmCPU, including the first three PCs, two PCs, and one PC as covariates, respectively; further, these QTL hit two (GS3 and SSG6), two (GS3 and GW6a), and one (GS3) known genes, respectively (Additional file 28: Figure S28), and overlapped with five, three, and one published QTL (Additional file 38: Table S15). When using FarmCPU including the first three PCs, we detected five and five QTL in the indica and japonica subpopulations, respectively, and these QTL hit two (GS3 and GW5) and one (TGW6) cloned genes, respectively (Additional file 29: Figure S29), overlapping with four and two of the published QTL (Additional file 38: Table S15). FarmCPU outperformed CMLM with respect to controlling inflation of $P$ values (Additional file 28: Figure S28e-h), identifying new QTL for rice grain length, and overlapping with known loci (Additional file 28: Figure S28a-d and Additional file 34: Table S5 and Additional file 38: Table S15). Unlike the FarmCPU, our association analysis for the rice grain size traits in Ho-LAMap employed GLM (Q) to try to avoid false negatives. There is no doubt that FarmCPU also could be integrated with Ho-LAMap in identifying new QTL because of its improved statistical power.

The era of deep-sequencing of vast arrays of germplasm has arrived and DNA sequencing has become quicker and cheaper. Thousands of rice accessions have been deepsequenced [23] and thousands of QTL on grain size (also other agronomic traits) have been mapped in rice (http:// archive.gramene.org/db/qtl/qtl_display?trait_category=Yield). However, how to rapidly identify the genes associated with agronomic traits utilizing these large amounts of sequence data and the linkage mapped QTL information in rice remains a challenge.

Ho-LAMap is an attractive method for rapid, costeffective identification of natural variations underlying QTL based on co-localization in multiple populations (Additional file 30: Figure S30 and Additional file 33: Notes S3). Coupled parents of populations having different genetic backgrounds can be divided into two classes similar to generating contrasting genetic pools for analysis of individual traits. Using the Ho index, we effectively distinguish a large number of background interference signals (i.e., false positives) from all (Additional file 12: Figure S12). Furthermore, candidate region association mapping conducted on SNPs located in the overlapping intervals of targeted QTL avoids false-positive loci located outside the critical region.

Deep sequencing of more than 3000 core rice collections with very broad genetic diversity and collected worldwide has been accomplished and our team has completed construction of approximately 100 recombinant inbred line populations based on accessions from our MCC panel. We expect that Ho-LAMap will facilitate gene isolation and rice breeding through molecular 
design by reducing the time for the construction of large NIL- $\mathrm{F}_{2}$ populations and fine mapping of QTL thus avoiding extremely laborious, time-consuming, and expensive fieldwork required for identification and cloning of agronomically important genes (Additional file 31: Figure S31) such as those for stress resistance.

\section{Methods}

Field planting and measurement of grain and yield traits

Rice plants were examined under natural field conditions at the two Experimental Stations of China Agricultural University, Beijing and Hainan, China. The planting density was $13.3 \mathrm{~cm}$ between plants in rows that were $23.3 \mathrm{~cm}$ apart. Field management, including irrigation, fertilizer application, and pest control, followed normal agricultural practices. Harvested rice grains were air-dried and stored at room temperature for at least 3 months before testing. Fully filled grains were used for measuring grain width, length, and weight. Ten randomly chosen grains from each plant were lined up width-wise along a Vernier caliper to measure grain width and then arranged length-wise to measure grain length. Grain weight was based on 200 grains and converted to 1000-grain weight.

\section{Plant material and backcross populations}

The extra large-grain rice accession SLG-1 (Oryza sativa L. ssp. japonica) and another two large-grain varieties, Haobuka and IRAT109, were selected from more than 7000 germplasm and used as desirable donor parents. The smallest grain accession, Chuanqi (ssp. indica), and two medium grain accessions, Nipponbare and Yuefu (ssp. japonica), were selected as recurrent parents. Four hybrid combinations (BILs from Nipponbare and SLG-1; CSSLs from Yuefu and IRAT109; $\mathrm{BC}_{1} \mathrm{~F}_{2}$ from Chuanqi and SLG-1; and $\mathrm{BC}_{1} \mathrm{~F}_{2}$ from Nippobare and Haobuka) were created by the six varieties.

The Nipponbare x SLG-1 cross: Four $\mathrm{BC}_{4} \mathrm{~F}_{2}$ populations for primary QTL mapping was constructed through selective backcrossing of lines which had large grain at each backcross generation. Three $\mathrm{BC}_{4} \mathrm{~F}_{3}$ populations for further QTL mapping were derived from some $\mathrm{BC}_{4} \mathrm{~F}_{2}$ plants without the known large-effect QTL (gs3 and $G W 2$ ). Using a similar strategy, we developed another two $\mathrm{BC}_{1} \mathrm{~F}_{2}$ populations: one from SLG-1 crossed with Chuanqi, the other one from the cross between Nipponbare and Haobuka.

The Yuefu x IRAT109 cross: Yuefu was crossed with IRAT109 and backcrossed with Yuefu for five generations, before selfing to produce $\mathrm{BC}_{5} \mathrm{~F}_{3}$ plants that were genotyped using 176 simple sequence repeat markers evenly distributed across all 12 rice chromosomes. Finally, we generated a fixed population of 271 CSSLs, with each containing an average three chromosome segments from the donor in the Yuefu genetic background.

\section{Selection of germplasm, sequencing and SNP identification}

The 506 worldwide accessions (Additional file 32: Table S1) contributed by the Chinese Academy of Agricultural Sciences included a mini-core collection of 248 accessions selected from a core collection of 932 accessions established from 61,470 O. sativa accessions preserved in the China National Crop Gene Bank [25], and 256 accessions selected based on isozyme diversity [48], used as parental lines in the international rice molecular breeding network, plus two accessions with the largest and smallest grains, respectively.

Genomic DNA was prepared from bulk-harvested leaves of a single young plant for each sampled accession by a modified CTAB method either at the International Rice Research Institute or at the Chinese Academy of Agricultural Sciences. Genomic DNA samples were then shipped to BGI-Shenzhen and were used to construct Illumina index libraries following the manufacturer's protocol. Following quality control, at least $3 \mu \mathrm{g}$ of genomic DNA of each sample was randomly fragmented by sonication and size-fractionated by electrophoresis. DNA fragments of approximately 500 bp were purified from each of 24 accessions and were labeled independently using distinct 6 bp nucleotide multiplex identifiers, followed by pooling prior to library construction for next generation sequencing. Each sequencing library was sequenced in six or more lanes on the HiSeq2000 platform and 90-bp paired-end reads were generated. Subsequently, the reads from each sample were extracted based on their unique nucleotide multiplex identifiers as 83 bp reads $(90-6-1$, where 1 is the ligation base “ $T$ "). To ensure high quality, raw data were filtered by deleting reads having adapter contamination or containing more than $50 \%$ low quality bases (quality value $\leq 5$ ).

The SNP data utilized for GWAS were generated as described in 'The 3000 rice genomes project' [23], and the SNP identification process was as follows:

- SNP calling for each sample were performed independently using the UnifiedGenotyper package in GATK with a minimum phred-scaled confidence threshold of 50, and a minimum phred-scaled confidence threshold for emitting variants at 10 . To ensure the quality of variant calling, the conditions for every site in a genome were set at $>20$ for mapping quality, $>50$ for variant quality, and $>2$ for the number of supporting reads for every base.

- Using IRGSP-1.0 as the reference, the 3000 sequenced genomes had an average depth of approximately $14 \times$, ranging from approximately $4 \times$ to greater than $60 \times$, and yielded a combined total of approximately $17 \mathrm{~TB}$ of high quality sequence data. Of the 3000 entries, 504 accessions used in this 
study had an average sequence depth of $14.9 \times$. Burrow-Wheeler alignment followed by variant calling using GATK identified approximately 10 million SNPs.

- The SNPs finally identified in this study were $3,585,228$ controlled by MAF $\geq 5 \%$ and missing rates $\leq 30 \%$.

\section{GWAS studies and candidate-region association mapping} GWAS of indica, japonica, and the full population were conducted using their corresponding genotype datasets. In each panel, only the SNPs with MAF > 5\% were used for association analyses. Three models - the naive model, GLM (Q), and CMLM - were used for association analysis [17]. The $\mathrm{Q}$ matrix and $\mathrm{K}$ matrix were estimated by STRUCTURE 2.0 and GAPIT function in R [49], respectively, and the $R$ version relies on the version of EMMA.

\section{QTL mapping and fine mapping}

A genetic map was constructed using MapMaker3.0/ EXP version 3.0 [50]. QTL analysis was performed by IciMapping3.1 [51] along with the composite interval mapping method, and the threshold was obtained by 1000 permutations. The substitution mapping strategy was used for fine mapping [52].

\section{Simulation analysis}

The significant SNPs within the QTL regions on both sides of physical position of each of seven wellcharacterized genes (i.e., GS3, GS5, GW5, GW8, OsLG3, GL7, and TGW6) and phenotypes of grain length, grain width, and grain weight were used in our simulation studies. The parents of biparental populations used for QTL mapping were randomly selected from the two tails of the population by extreme trait performance. Since there are two alleles for each SNP, say $A$ and $a$, let $p_{H}$ denote the allele frequency of $A$ in one tail of the population with high trait performance, and $p_{L}$ denote the allele frequency of $A$ in the other tail with low trait performance. That is to say, the allele frequency of $A$ for one parent was $p_{H}$, and that for the other parent was $p_{L}$. If the SNP locus is polymorphic between two parents, it could be significantly associated with the trait of interest. The power of Ho-LAMP method can be estimated as $p_{H}$ $\left(1-p_{L}\right)+\left(1-p_{H}\right) p_{L}$. To evaluate the effect of population structure on the power of Ho-LAMP method, we conducted simulation experiments for all 504 rice cultivars, Japonica cultivars, and Indica cultivars as groups. We also tried percentages of $0.05,0.10$, and 0.15 to select sub-populations with extreme phenotypic performance on both sides. Results were largely similar; therefore, 0.05 was used as the percentage cut-off to determine the size of the two tails of the population.
Simulation of cross number also used significant SNPs of some of these well-characterized genes (i.e., GW8, GS3, and TGW6). Firstly, 504 accessions were divided into two parts by functional alleles of each gene, called A and a. Randomly selected similar numbers of varieties from each part were used to calculate the Ho-index for functional alleles in the candidate region. We simulated 1000 repeats and counted the times when only the functional allele had the maximum Ho-index, denoted as one time and three replicates for each gene. We evaluated the power as the proportion of detecting the target gene successfully in 1000 replications of simulation. Finally, we set different cross numbers (2-50) to carry out the simulation and defined the ideal number of crosses for each gene. The same process was applied to the indica and japonica populations, respectively.

\section{Vector construction and plant transformation}

Full-length cDNA of OsLG3 was amplified from the first-strand cDNA of IRAT109 with specific primers to generate the overexpression construct (Additional file 33: Table S17) using PrimeSTAR HS DNA Polymerase (TaKaRa). The sequence-confirmed PCR fragment was digested with Kpn I and Pac I, and inserted into the vector pMDC32 under the control of the Cauliflower Mosaic virus (CaMV) 35S promoter.

To generate the RNA interference (RNAi) construct, a fragment targeting the 294 bp coding region of OsLG3 was amplified with specific primers P3 and P4, digested with Kpn I and BamH I and then Spe I and Sac I sites, and subsequently inserted into the pTCK303 vector as previously described [53]. Primers used in these experiments are listed in Additional file 33: Table S17. To generate transgenic plants, the constructs were transformed into Nipponbare by Agrobacterium-mediated transformation [54]. Positive transgenic plants were selected by germinating transgenic seeds on $1 / 2$ MS medium containing $50 \mathrm{mg} / \mathrm{L}$ hygromycin (Roche, Germany).

\section{RNA extraction and expression analysis}

For expression analysis, rice leaves were flash-frozen in liquid nitrogen, total RNAs were extracted using RNAiso Plus (TaKaRa) according to the manufacturer's instructions; $4 \mu \mathrm{g}$ of the DNase-treated RNA were reverse transcribed using M-MLV reverse transcriptase (TaKaRa). The resulting cDNA samples were diluted five times and used as templates for PCR.

qRT-PCR was performed in an Applied Biosystems 7500 Real Time PCR system (ABI, USA) using SYBR Premix Ex Taq $^{\mathrm{Tm}}$ II (TaKaRa) as previously described [55, 56]. The gene-specific primers used for real-time PCR are listed in Additional file 33: Table S16. qRT-PCR was performed in triplicate for each sample, and the Actin1 
gene was used as the internal reference for data normalization using the $2^{-\Delta \Delta t}$ method [57].

\section{Sub-cellular localization of OsLG3 and transactivation activity assay}

To generate the 35S::OsLG3-GFP construct, the full-length ORF of OsLG3 without the terminal codon was amplified with the corresponding primers (Additional file 33: Table S17). The amplified fragments were digested with Kpn I and Pac I, and then cloned into the pMDC83 vector and fused with the GFP reporter gene driven by the CaMV 355 promoter. The recombinant constructs of the 35S:OsLG3-GFP fusion and GFP alone were transiently transfected into onion epidermal cells, using a PDS-1000/ He system (Bio-Rad, USA) at 1100 psi. After incubation at $25{ }^{\circ} \mathrm{C}$ for $24 \mathrm{~h}$, the fluorescence signal was examined through a confocal laser scanning microscope FV1000 (Olympus, USA) with excitation at $488 \mathrm{~nm}$ and emission at $525 \mathrm{~nm}$.

For the transactivation assay, plasmids pGBKT7-OsLG3 (full length coding region of OsLG3), pGBKT7-OsLG3-N (N-terminal of OsLG3, amino acids 1-108), pGBKT7OsLG3-C (C-terminal of OsLG3, amino acids 109-209) were constructed. The pGBKT7 vector was used as a negative control and pGBKT7-53 was used as a positive control. These constructs were introduced into yeast strain AH109 by LiAc-mediated yeast transformations, and screened on selective medium plates without tryptophan (SD/-Trp). The PCR-verified transformants were transferred to SD medium without tryptophan/histidine/ adenine (SD/-Trp/-His/-Ade) for 3 days. Transactivation activities were performed according to the in vivo agar plate assay $(\mathrm{x}-\alpha-\mathrm{gal}$ in medium).

\section{Histological analysis}

Milled rice grains for scanning electron microscopy were transversely cut in the middle with a knife and coated with gold under vacuum conditions. The morphology of starch granules in the belly part of the endosperm was examined with a scanning electron microscope (Hitachi, S-570, China Atomic Energy Research Institute) at an accelerating voltage of $12 \mathrm{kV}$. The analysis was based on at least three biological replications of mounted specimens. All procedures were carried out according to the manufacturer's protocol.

\section{Nucleotide diversity analysis and minimum spanning tree} The average nucleotide diversities $(\pi)$ of indica, temperate japonica, tropical japonica, and wild rice subpopulations were estimated in non-overlapping $10 \mathrm{~kb}$ windows using an in-house Perl script; missing data positions were included, with a modification of population size [58].
Five hundred and four diverse cultivated rice and 15 $O$. rufipogon accessions from around the world (Additional file 32: Table S1) were used to construct a minimum spanning tree for OsLG3. Arlequin version 3.5 [59] was used to define the haplotypes and calculate the minimum spanning tree among haplotypes. Arlequin's distance matrix output was used in Hapstar-0.7 [60] to draw a minimum spanning tree.

\section{Phylogenetic and genetic interaction analysis}

The phylogenetic tree of 480 varieties was constructed based on SNPs and indels that were proven FNPs (listed in Additional file 32: Table S1) by MAGE 6.0. The EvolView [61] online tool was used for visualizing the phylogenetic tree. Grain length was measured by Vernier calipers and the violin map for genetic interaction analysis was constructed in R. Multiple comparisons were made by Tukey' honest significant difference in $\mathrm{R}$. Landraces and raw data are listed in Additional file 32: Table S1.

\section{Additional files}

\begin{abstract}
Additional file 1: Figure S1. Geographic origins of 504 rice accessions. (a) Geographic origins of worldwide rice cultivars. The smaller pie charts on the world map correspond to the country-specific distribution of subpopulations sampled (note: China was divided into several major rice growing regions). The size of the pie chart is proportional to the sample size and colors within each pie chart are reflective of the percentage of samples in each subpopulation. Seeds representing each subpopulation are displayed with and without hull in the center, with $5 \mathrm{~mm}$ scale bar. (b) Geographic distribution of these accessions. Number of countries sampled in each geographical region are indicated on top of the bars. (PDF 250 kb)
\end{abstract}

Additional file 2: Figure S2. Frequency distribution of grain length in the mini core collection (MCC population). (PDF $117 \mathrm{~kb}$ )

Additional file 3: Figure S3. Comparison of GWAS of grain length in the full population using LM model (a), CMLM model (b), and GLM (Q) model (c). In these Manhattan plots, for the significant loci identified, known loci are shown in red. Blue horizontal solid lines indicate the genome-wide significance threshold. (PDF $114 \mathrm{~kb}$ )

Additional file 4: Figure S4. Correlation between grain length and population structure in the natural population and frequency distribution between two genotypes in subpopulation about GS3, GS5, TGW6, GW8, and OsLG3. (a) Distribution of germplasms with different grain length according to PC structure. (b) Frequency distribution between two genotypes in subpopulation about GS3, GS5, TGW6, GW8, and OsLG3. The rate of each haplotype under two sub population divided by $\mathrm{Q}$ for these genes. Ind, indica; jap, japonica. (PDF $246 \mathrm{~kb}$ )

Additional file 5: Figure S5. Effect of Q structure (indica and japonica) for grain length. (a) Comparison of grain length between big-grain-haplotype and small-grain-haplotype when Q structure exists. (b) Comparison of grain length between big-grain-haplotype and small-grain-haplotype when the phenotype variation from Q structure was removed. Ind, indica; jap, japonica. Data are means \pm SEM. Letters indicate a significant difference at $P<0.01$ $(n=3)$ by the Student's $t$-test. (PPTX $362 \mathrm{~kb})$

Additional file 6: Figure S6. Size of association regions about GS3, TGW6, GW8, and OsLG3. Manhattan plots in candidate region of three known genes and OSLG3 in different models. The red points indicate their SNPs within gene region, respectively. The horizontal full lines indicate the genome-wide significance threshold (0.05/n). (PDF $311 \mathrm{~kb}$ ) 
Additional file 7: Figure S7. Grains from six parents. Scale bar, $5 \mathrm{~mm}$. (PDF $109 \mathrm{~kb}$ )

Additional file 8: Figure S8. Frequency distribution of variation of grain traits in biparental populations derived from NIP and SLG. (a-d) Phenotype variants of grain traits for populations 07DH010, 07DH011, 07DH013, and 07DH014, respectively. (PDF $103 \mathrm{~kb}$ )

Additional file 9: Figure S9. QTL detected in the four crosses derived from six varieties. (a) Distribution of grain shape QTL and genes on genetic linkage map. Blue strip refers to grain length QTL, green to 1000-grain weight and grain width QTL, red to grain thickness QTL and arrows to cloned genes. (b) Heat map for effect of grain length QTL mapped by four linkage populations. Rows of the heat map correspond to the $14 \mathrm{QTL}$ for grain length. NIP, Nipponbare; SLG, SLG-1; CQ, Chuanqi; YF, Yuefu; IR109, IRAT109; HBK, Haobuka. (PDF 224 kb)

Additional file 10: Figure S10. Fine mapping of $9 G L 3-3$. White bars represent chromosomal segments for NIP homozygote, black for SLG homozygote, and grille for heterozygote, respectively. Progeny testing was used to confirm the genotypes at the qGL3-3 locus. S, segregation; $\mathrm{D}$, desegregation. (PDF $78 \mathrm{~kb})$

Additional file 11: Figure S11. Correlations between the grain traits in the MCC panel (a) and 07DH014 population (b). Number in blue, phenotypic correlation in $r^{2}$ between traits. (PDF $124 \mathrm{~kb}$ )

Additional file 12: Figure S12. Simplified scheme for application of Ho-LAMap to rice. (a) We cross diverse founder varieties (i.e., variety that is significantly different to the reference parent on grain traits) with reference parent (usually has small grain). The founder varieties are deep sequenced by the second-generation sequencing platforms, as in Additional file 30: Figure S30. In several crosses that have detected targeted QTL, the majority of SNPs between the QTL interval will segregate in a 1:1 founder varieties: reference parent ratio. However, the SNP responsible for the change of phenotype is the same in all founder parents, which can detect the targeted QTL. If we define the Ho (observed heterozygosity per locus) index as the ratio between the number of heterozygous crosses corresponding to each SNP locus and the total number of crosses which have detected targeted QTL, we expect this index would equal 1 near the causal SNP and 0.5 for the unlinked loci. (b, d) Candidate region association mapping. The brown horizontal dashed lines indicate the genome-wide significance threshold. The red points indicate significant loci within candidate gene. (c) Ho index plots for the target QTL. Red regression lines were obtained by averaging SNP indices from a sliding window analysis. (PDF $105 \mathrm{~kb}$ )

Additional file 13: Figure S13. Simulation reveals effectiveness of Ho-LAMap in different subgroups for several known genes (such as GW8, TGW6, etc.) about grain size when using Ho-LAMap. The subgroups contain indica and japonica population. (PPTX $671 \mathrm{~kb}$ )

Additional file 14: Figure S14. Simulation reveals the cross number needed for three known genes for grain size when using Ho-LAMap. Simulation of crosses number also used significant SNPs of some of these well characterized genes (i.e., TGW6 (a), GS3 (b), and GW8 (c)). The x-axis value indicates cross number. Full pop, the full population; indica and japonica, the indica and japonica subgroup. We evaluated the power as the probability of detecting the target gene successfully in 1000 replications of the simulation. (PDF $227 \mathrm{~kb}$ )

Additional file 15: Figure S15. Identification of the causal SNPS of QTL qGL3-2 using Ho-LAMap. We also used Ho-LAMap to clone OsLG3b from $q G L 3-2$, a new gene for grain length, which encodes a MADS-box transcription factor. The top is Manhattan plot for candidate region association mapping for QTL region of qGL3-2; the middle correspond to Ho index plots for QTL region of qGL3-2. The bottom correspond to candidate gene (OsLG3b), the green dashed lines label the region for significant signal. (PDF $82 \mathrm{~kb}$ )

Additional file 16: Figure S16. Nucleotide diversity analysis for the promoter region and CDS of OsLG3. (PDF $59 \mathrm{~kb}$ )

Additional file 17: Figure S17. OsLG3 expression levels in organs from NIP and NIL(SLG) plants. FL, flag leaf at the heading date; S, stem; H4B and $\mathrm{H} 2 \mathrm{~B}$, hulls at 4 and 2 days before heading; $\mathrm{H} 4 \mathrm{~A}$, hull at 4 days after heading; $5 \mathrm{E}$ and $10 \mathrm{E}$, endosperm at 5 and 10 days after fertilization; $n=3$. Data are given as mean \pm SEM. (PDF $84 \mathrm{~kb}$ )
Additional file 18: Figure S18. Phenotypes of grain in three OsLG3-overexpressing lines and three RNAi-OsLG3 lines. (a, d) Grain length of the transgenic and control lines $(n=30)$. (b, e) 1000-grain weight in the transgenic and control lines $(n=30)$. ( $(c, f)$ Relative expression levels of OsLG3 in young panicles of the transgenic and control lines were detected by qPCR, with data normalized to OsActin 1 levels $(n=3)$. All data in $c$, $f-h$, and $\mathrm{j}-\mathrm{l}$ are presented as means \pm SEM. ${ }^{* *} P<0.01$, Student's $t$ test. (PDF $77 \mathrm{~kb}$ )

Additional file 19: Figure S19. A field trial of NIP and NIL(SLG) plants. (a) The morphology of the NIL plants. Scale bar, $10 \mathrm{~cm}$. (b) Plant height. (c) Tiller number. (d) Number of grains per panicle. (e) Heading date. All phenotypic data in b-e were measured from plants grown with $20 \times 20 \mathrm{~cm}$ spacing in paddies under normal cultivation conditions. Data represent mean \pm SEM. $(n=30)$. Student's $t$ tests were used to generate $P$ values. (PDF $111 \mathrm{~kb}$ )

Additional file 20: Figure S20. OsLG3 regulates grain length by changing cell division patterns. (a) The grains of NIP and NIL(SLG) plants (top) and scanning electron microscope images of the outer glume (middle) and inner epidermal cells of the lemma (bottom) before anthesis. Scale bars, $5 \mathrm{~mm}$ (white; top) and $300 \mu \mathrm{m}$ (yellow; middle and bottom). (b) Cell length of outer epidermal cells in the longitudinal direction in a $(n=12)$. (c) Cell width of outer epidermal cells in the longitudinal direction in (a) $(n=12)$. (d) Total cell number of outer epidermal cells in the longitudinal direction. (e) Cell length of inner epidermal cells in the longitudinal direction in (a) $(n=12)$. (f) Cell width of inner epidermal cells in the longitudinal direction in (a) $(n=12)$. (g) Total cell number of inner epidermal cells in the longitudinal direction. All data represent means \pm SEM. ${ }^{*} P<0.05,{ }^{*} P<0.01$, Student's $t$ test. (PDF $134 \mathrm{~kb}$ )

Additional file 21: Figure S21. The effect of OsLG3 on the expression of genes involved in cell cycle. The expression analysis was conducted using 7-cm long young panicles. OsActin1 was used as the control and the values of expression levels in Nipponbare were set to $1(n=3)$. Data are given as mean \pm SEM. Student's $t$-test was used to generate the $P$ values; ${ }^{*} P<0.05$, ${ }^{*} P<0.01$, respectively. (PDF $135 \mathrm{~kb}$ )

Additional file 22: Figure S22. OSLG3 does not affect grain quality. (a) Scanning electron microscopy images are transverse sections of starch granule of NIP and NIL(SLG). Scale bars, $50 \mu \mathrm{m}$ (green line). (b) Comparison of brown grains NIP and NIL(SLG). Scale bar, $2 \mathrm{~cm}$. (c) The ratio of grain length to width $(n=30)$. (d) Percentage of grain with chalkiness (\%) $(n=6)$. (e) Square of chalky endosperm $(\%)(n=6)$. Data are given as means \pm SEM. Student's $t$-test was used to generate the $P$ values; ${ }^{*} P<0.05,{ }^{* *} P<0.01$, respectively. (PPTX $405 \mathrm{~kb}$ )

Additional file 23: Figure S23. Overexpression of OsLG3 in NIP has large effect on grain length and cell number. (a) The mature rice grain images of NIP (left) and over-expression lines (right), OE-3, OE-4, and $\mathrm{OE}-5$, respectively. The scanning electron microscope images are the outer glume epidermal cells of the lemma from the spikelet hulls of NIP (left) and over-expression plant (right) before anthesis, respectively. Scale bars, $5.0 \mathrm{~mm}$ (white line) and $300 \mu \mathrm{m}$ (yellow line), respectively. (b) Grain length. (c-e) Cell length of outer epidermal cells in the longitudinal direction in a $(n=12)$. ( $f-h)$ Total cell number of outer epidermal cells in the longitudinal direction $(n=12)$. All phenotypic data in $b-h$ were measured from plants grown with $15 \times 20 \mathrm{~cm}$ spacing in paddies under normal cultivation conditions. All data represent means \pm SEM. ${ }^{*} P<0.05$, ${ }^{*} P<0.01$, Student's $t$ test. (PDF $107 \mathrm{~kb}$ )

Additional file 24: Figure S24. Effect of RNAi-OSLG3 in NIP on grain length. RNAi-OsLG3 in NIP has large effect on grain length. (a) The mature rice grain images are control (left) and RNAi-1, RNAi-2, and RNAi-3, respectively (right). The scanning electron microscope images are the outer glume epidermal cells of the lemma from the spikelet hulls of NIP (left) and RNAi-OsLG3 plant (right) before anthesis, respectively. Scale bars, $5.0 \mathrm{~mm}$ (white line) and $300 \mu \mathrm{m}$ (yellow line), respectively. (b) Grain length. (c-e) Cell length of outer epidermal cells in the longitudinal direction in a $(n=12)$. ( $f-h)$ Total cell number of outer epidermal cells in the longitudinal direction $(n=12)$. All phenotypic data in $\mathrm{b}-\mathrm{h}$ were measured from plants grown with $15 \times 20 \mathrm{~cm}$ spacing in paddies under normal cultivation conditions. All data represent means \pm SEM. ${ }^{*} P<0.05$, ${ }^{* *} P<0.01$, Student's $t$ test. (PDF $89 \mathrm{~kb}$ )

Additional file 25: Figure S25. OsLG3 expression activity was monitored by POsLG3:GUS transgene expression. Histochemical analysis 
of GUS activity in root (a), stem (b), sheath (c), leaf (d), and the developing panicles (e). Scale bar, $1 \mathrm{~cm}$. (PDF $90 \mathrm{~kb}$ )

Additional file 26: Figure S26. Genetic interactions among OsLG3, GS3, GW8, and TGW6. (a) Varieties were categorized by allelic variations of OsLG3, GS3, and TGW6. (b) Varieties were categorized by allelic variations in the functional SNP of OsLG3, GS3, and GW8. Grain length was measured by Vernier calipers, and the violin map was constructed in R. Multiple comparisons were done by Tukey' HSD in R. Landraces and raw data were listed in Additional file 32: Table S1. (PDF $143 \mathrm{~kb}$ )

Additional file 27: Figure S27. The spectrum of allele frequencies between landrace and improved varieties at the causal polymorphisms of GS3, OsLG3, GW8, and TGW6 during modern breeding in full population (a) and japonica subgroup (b). LAN, landrace; IMP, improved variety. (PDF $199 \mathrm{~kb}$ )

Additional file 28: Figure S28. Comparison of GWAS of grain length in the full population using MLM (a), FarmCPU included the first three PCs (b), two PCs (c), and one PC (d) as covariates. In these Manhattan plots, for the significant loci identified, five SNPs within a 200-kb range of known genes are marked as significant loci re-identified. Green horizontal solid lines in (b), (c), and (d) indicate the Bonferroni-corrected significant threshold $(0.01 / n)$. However, the genome-wide significance threshold of all methods in this study adopts the Bonferroni-corrected threshold with $0.05(0.05 / \mathrm{n})$ (see Additional file 38: Table S15). Quantile-quantile plots of these models are shown in e, $\mathrm{f}, \mathrm{g}$, and $\mathrm{h}$, respectively. (PDF $261 \mathrm{~kb}$ )

Additional file 29: Figure S29. Comparison of GWAS of grain length in the full population (a), indica (b), and japonica (c) subpopulations using FarmCPU included the first three PCs as covariates. In these Manhattan plots, for the significant loci identified, five SNPs within a 200-kb range of known genes are marked as significant loci re-identified. Green horizontal solid lines in (a), (b), and (c) indicate the Bonferroni-corrected significant threshold $(0.01 / n)$. However, the genome-wide significance threshold of al methods in this study adopts the Bonferroni-corrected threshold with 0.05 (0.05/n) (see Additional file 38: Table S15). Quantile-quantile plots in these three populations are shown in $\mathrm{d}$, e, and f, respectively. (PDF $251 \mathrm{~kb}$ )

Additional file 30: Figure S30. Proposed strategy of gene discovery by Ho-LAMap. The number 1 and 2 in the red cycle indicate two pathways for gene discovery by Ho-LAMap. At the first way, a lot of QTL had been co-localized in many crosses by predecessors. Therefore, we re-sequenced parents of those crosses and isolated gene from the target QTL via Ho-LAMap. For the second way, we obtained the QTL region by GWAS and selected extreme materials to construct several $F_{2}$ or recombinant inbred line populations according to peak SNP and phenotypes. Then, we could ascertain several crosses that can map the same QTL by linkage mapping and rapidly isolated gene from QTL by Ho-LAMap directly. (PDF $19 \mathrm{~kb}$ )

Additional file 31: Figure S31. Identification of the causal SNPS of QTL sdl using Ho-LAMap. The top is Manhattan plot for candidate region association mapping for QTL region of $s d 1$; the middle corresponds to $\mathrm{Ho}$ index plots for QTL region of sd1. The bottom corresponds to candidate gene (sd1), the green dashed lines label the region for significant signal. (PDF $175 \mathrm{~kb}$ )

Additional file 32: Table S1. Information of Oryza sativa L. varieties and wild rice on variety name, geographic source, stratification referred by STRUCTURE, the integrated stratification, grain lengths, and allelic variations of grain length-related genes. (XLSX $115 \mathrm{~kb}$ )

Additional file 33: Notes S1. Natural population and linkage mapping (Additional file 2: Figure S2 and Additional file 11). Notes S2. Fine mapping of qGL3-3 (Additional file 10). Notes S3. Ho-LAMap - a potential method for validating association peak. Table S2. Summary of the taxa and source of 506 varieties of Oryza sativa L. Table S3. Environments used to evaluate association and linkage populations. Table $\mathbf{S 4}$. The heritability of grain traits in MCC1 Panel. Table S9. Summary of causal allele and type of variation about several grain size genes. Table S10. Grain phenotypes of six different parents. Table S11. Quantitative trait loci mapped in the four populations. Table S12. Simulation reveals the cross number need for several known genes (such as TGW6, GS3) about grain size when using Ho-LAMap. Table S13. OsLG3 polymorphisms associated with grain length in the MCC panel. Table S14. Comparison of polymorphisms between three large grain parents and three small grain parents. Table S16. Primers used for fine mapping and sequencing. Table S17. Primers used for DNA constructs and transcript analysis. (DOCX $73 \mathrm{~kb}$ )
Additional file 34: Table S5. Genome-wide significantly associated loci $\left(-\log _{10}(P) \geq 7.9\right)$ with $\mathrm{GL}$ in full population, indica and japonica population using CMLM model and QTL co-localized with linkage mapping. (XLSX 9 kb)

Additional file 35: Table S6. Genome-wide significantly associated loci with $\mathrm{GL}$ in full population using GLM (Q) model and QTL co-localized with linkage mapping. (XLSX $15 \mathrm{~kb}$ )

Additional file 36: Table S7. Genome-wide significantly associated loci with GL in indica population using GLM (Q) model and QTL co-localized with linkage mapping. (XLSX $11 \mathrm{~kb}$ )

Additional file 37: Table S8. Genome-wide significantly associated loci with GL in japonica population using GLM (Q) model and QTL co-localized with linkage mapping. (XLSX $14 \mathrm{~kb}$ )

Additional file 38: Table S15. Genome-wide significantly associated loci with GL in full population, indica population, and japonica population using FarmCPU and QTL co-localized with linkage mapping. (XLSX $13 \mathrm{~kb}$ )

Additional file 39: Supporting data. (XLSX $79 \mathrm{~kb})$

\section{Acknowledgments}

We thank Professor Robert A McIntosh (University of Sydney) for critical reading and suggested revision of the manuscript, Yan Liu for QTL analysis, Yanfa Chen and Haifeng Guo for preparing samples, and Jianyin Xie and Yan Zhao for help with data analysis.

\section{Funding}

This work was supported by the China National Key Technologies R\&D Program (2015BAD02B01, 2016YFD0100101, and 2013BAD01B02-15), 948 project of the MOA (2011-G2B and 2011-G1 (2)-25), Key Program of GuangXi Academy of Agricultural Sciences (Gui nong ke 2016JZ05), Fund of Chairman of Guangxi autonomous region (1517-03), and Basal Research Fund of GuangXi Academy of Agricultural Sciences (2015YT14).

\section{Availability of data and materials}

The data on 504 accessions (Additional file 32: Table S1) in our study are a part of the 3000 rice genomes project. All SNPs and indels of the accessions in our study can be found at http://www.rmbreeding.cn/snp3k. We re-sequenced the OsLG3 region of 27 wild rice accessions (Additional file 32: Table S1). Sequence data for another 10 wild rice accessions were obtained from ftp://rice:ricedownload@ public.genomics.org.cn/BGl/rice. All data generated or analyzed during this study are included in this published article and its supplementary information files. Raw data can be found in Additional file 39.

\section{Authors' contributions}

$J Y$ and ZiL designed the research, and together with $H X$ and $X Z$, performed most of experiments and analyzed the data. HL conducted the simulation analysis. GY and QZ constructed the genetic populations. JM, $Y P, Z Z$, and $X W$ performed part of the experiments. WW, ZT, YG, and ZhL conceived and supervised the project. JY, XF, HZ, JL, and ZiL conceived the experiment and wrote the manuscript. All authors read and approved the final manuscript.

\section{Competing interests}

The authors declare that they have no competing interests.

\section{Consent for publication}

Not applicable.

Ethics approval and consent to participate Not applicable.

\section{Publisher's Note}

Springer Nature remains neutral with regard to jurisdictional claims in published maps and institutional affiliations.

\section{Author details}

'Key Laboratory of Crop Heterosis and Utilization, Ministry of Education/ Beijing Key Laboratory of Crop Genetic Improvement, China Agricultural University, Beijing 100193, China. ${ }^{2}$ Institute of Crop Sciences, Chinese Academy of Agricultural Sciences, Beijing, China. ${ }^{3}$ Institute of Genetics and 
Developmental Biology, Chinese Academy of Sciences, Beijing, China. ${ }^{4}$ Rice Research Institute, Guangxi Academy of Agricultural Sciences, Nanning, Guangxi, China.

Received: 7 November 2016 Accepted: 10 March 2017

Published online: 06 April 2017

\section{References}

1. Shomura A, Izawa T, Ebana K, Ebitani T, Kanegae H, Konishi S, et al. Deletion in a gene associated with grain size increased yields during rice domestication. Nat Genet. 2008:40(8):1023-8.

2. Huang $R$, Jiang L, Zheng J, Wang T, Wang H, Huang Y, et al. Genetic bases of rice grain shape: so many genes, so little known. Trends Plant Sci. 2012;18(4):218-26

3. Mao H, Sun S, Yao J, Wang C, Yu S, Xu C, et al. Linking differential domain functions of the GS3 protein to natural variation of grain size in rice. Proc Natl Acad Sci U S A. 2010;107(45):19579-84

4. Song XJ, Huang W, Shi M, Zhu MZ, Lin HX. A QTL for rice grain width and weight encodes a previously unknown RING-type E3 ubiquitin ligase. Nat Genet. 2007:39(5):623-30.

5. Zhang $X$, Zhang $H$. Rare allele of OsPPKL1 associated with grain length causes extra-large grain and a significant yield increase in rice. Proc Natl Acad Sci U S A. 2012;109(52):21534.

6. Ishimaru K, Hirotsu N, Madoka Y, Murakami N, Hara N, Onodera H, et al. Loss of function of the IAA-glucose hydrolase gene TGW6 enhances rice grain weight and increases yield. Nat Genet. 2013;45(6):707-11.

7. Wang S, Wu K, Yuan Q, Liu X, Liu Z, Lin X, et al. Control of grain size, shape and quality by OsSPL16 in rice. Nat Genet. 2012;44(8):950.

8. Frary A, Nesbitt TC, Frary A, Grandillo S, Knaap E, Cong B, et al. fw2.2: A quantitative trait locus key to the evolution of tomato fruit size. Science. 2000;289(5476):85-8

9. Li Y, Fan C, Xing Y, Yun P, Luo L, Yan B, et al. Chalk5 encodes a vacuolar H +-translocating pyrophosphatase influencing grain chalkiness in rice. Nat Genet. 2014;46(4):398-404.

10. Ma Y, Dai X, Xu Y, Luo W, Zheng X, Zeng D, et al. COLD1 confers chilling tolerance in rice. Cell. 2015;160(6):1209-21.

11. Liu Y, Wu H, Chen H, Liu Y, He J, Kang H, et al. A gene cluster encoding lectin receptor kinases confers broad-spectrum and durable insect resistance in rice. Nat Biotech. 2015;33(3):301-5.

12. Huang $X$, Wei $X$, Sang $T$, Zhao Q, Feng Q, Zhao Y, et al. Genome-wide association studies of 14 agronomic traits in rice landraces. Nat Genet. 2010;42(11):961-7.

13. Huang X, Zhao Y, Wei X, Li C, Wang A, Zhao Q, et al. Genome-wide association study of flowering time and grain yield traits in a worldwide collection of rice germplasm. Nat Genet. 2012;44(1):32-9.

14. Tian F, Bradbury PJ, Brown PJ, Hung H, Sun Q, Flint-Garcia S, et al. Genome-wide association study of leaf architecture in the maize nested association mapping population. Nat Genet. 2011:43(2):159-62.

15. Jia G, Huang X, Zhi H, Zhao Y, Zhao Q, Li W, et al. A haplotype map of genomic variations and genome-wide association studies of agronomic traits in foxtail millet (Setaria italica). Nat Genet. 2013:45(8):957-61.

16. Kump KL, Bradbury PJ, Wisser RJ, Buckler ES, Belcher AR, Oropeza-Rosas MA, et al. Genome-wide association study of quantitative resistance to southern leaf blight in the maize nested association mapping population. Nat Genet. 2011;43(2):163-8.

17. Atwell S, Huang YS, Vilhjalmsson BJ, Willems G, Horton M, Li Y, et al. Genome-wide association study of 107 phenotypes in Arabidopsis thaliana inbred lines. Nature. 2010;465(7298):627-31.

18. Payseur BA, Place M. Prospects for association mapping in classical inbred mouse strains. Genetics. 2007:175(4):1999-2008.

19. Nemri A, Atwell S, Tarone AM, Huang YS, Zhao K, Studholme DJ, et al. Genome-wide survey of Arabidopsis natural variation in downy mildew resistance using combined association and linkage mapping. Proc Natl Acad Sci. 2010;107(22):10302-7.

20. Wang $Q$, Xie W, Xing H, Yan J, Meng X, Li X, et al. Genetic architecture of natural variation in rice chlorophyll content revealed by a genome-wide association study. Mol Plant. 2015;8(6):946-57.

21. Lou Q, Chen L, Mei H, Wei H, Feng F, Wang P, et al. Quantitative trait locus mapping of deep rooting by linkage and association analysis in rice. $J$ Exp Bot. 2015;66(15):4749-57.
22. Crowell S, Korniliev P, Falcão A, Ismail A, Gregorio G, Mezey J, et al. Genome-wide association and high-resolution phenotyping link Oryza sativa panicle traits to numerous trait-specific QTL clusters. Nat Commun. 2016;7:10527.

23. The 3,000 rice genomes project. GigaScience. 2014;3(1):7

24. Alexandrov N, Tai S, Wang W, Mansueto L, Palis K, Fuentes RR, et al. SNP-Seek database of SNPs derived from 3000 rice genomes. Nucleic Acids Res. 2015;43(Database issue):D1023.

25. Zhang H, Zhang D, Wang M, Sun J, Qi Y, Li J, et al. A core collection and mini core collection of Oryza sativa L. in China. Theor Appl Genet. 2011:122(1):49-61.

26. Yu SB, Xu WJ, Vijayakumar CH, Ali J, Fu BY, Xu JL, et al. Molecular diversity and multilocus organization of the parental lines used in the International Rice Molecular Breeding Program. Theor Appl Genet. 2003;108(1):131-40.

27. Yu J, Pressoir G, Briggs WH, Bi IV, Yamasaki M, Doebley JF, et al. A unified mixed-model method for association mapping that accounts for multiple levels of relatedness. Nat Genet. 2006;38(2):203-8.

28. Zhang Z, Ersoz E, Lai C-Q, Todhunter RJ, Tiwari HK, Gore MA, et al. Mixed linear model approach adapted for genome-wide association studies. Nat Genet. 2010;42(4):355-60.

29. Zhao K, Tung CW, Eizenga GC, Wright MH, Ali ML, Price AH, et al. Genome-wide association mapping reveals a rich genetic architecture of complex traits in Oryza sativa. Nat Commun. 2011;2:467.

30. Itoh Jl, Nonomura Kl, Ikeda K, Yamaki S, Inukai Y, Yamagishi H, et al. Rice plant development: from zygote to spikelet. Plant Cell Physiol. 2005;46(1):23.

31. Berckmans B, De VL. Transcriptional control of the cell cycle. Curr Opin Plant Biol. 2009;12(5):599-605

32. Weigel D. The APETALA2 domain is related to a novel type of DNA binding domain. Plant Cell. 1995;7(4):388-9.

33. Hao D, Ohme-Takagi M, Sarai A. Unique mode of GCC box recognition by the DNA-binding domain of ethylene-responsive element-binding factor (ERF domain) in plant. J Biol Chem. 1998;273(41):26857-61.

34. Asano K, Matsuoka M. Artificial selection for a green revolution gene during japonica rice domestication. Proc Natl Acad Sci U S A. 2011;108(27):11034-9.

35. Kovach MJ, Sweeney MT, McCouch SR. New insights into the history of rice domestication. Trends Genet. 2007:23(11):578-87.

36. Li N, Li Y. Signaling pathways of seed size control in plants. Curr Opin Plant Biol. 2016:33:23-32.

37. Sharma S, Tripathy S, Biswal J. Origin of asian cultivated rice and its ecotypic differentiation. Indian J Genet Plant Breed. 1997:57(4):339-60.

38. Chang T-T. The origin, evolution, cultivation, dissemination, and diversification of Asian and African rices. Euphytica. 1976;25(1):425-41.

39. Londo JP, Chiang Y-C, Hung K-H, Chiang T-Y, Schaal BA. Phylogeography of Asian wild rice, Oryza rufipogon, reveals multiple independent domestications of cultivated rice. Oryza sativa Proc Natl Acad Sci. 2006;103(25):9578-83.

40. Huang X, Kurata N, Wei X, Wang Z, Wang A, Zhao Q, et al. A map of rice genome variation reveals the origin of cultivated rice. Nature. 2012:490:497-501.

41. Civáň P, Craig H, Cox CJ, Brown TA. Three geographically separate domestications of Asian rice. Nat Plants. 2015;1:15164.

42. Li C, Zhou A, Sang T. Rice domestication by reducing shattering. Science. 2006:311(5769):1936-9.

43. Tan L, Li X, Liu F, Sun X, Li C, Zhu Z, et al. Control of a key transition from prostrate to erect growth in rice domestication. Nat Genet. 2008;40(11):1360-4.

44. Zhu B-F, Si L, Wang Z, Zhu YZJ, Shangguan Y, Lu D, et al. Genetic control of a transition from black to straw-white seed hull in rice domestication. Plant Physiol. 2011;155(3):1301-11.

45. Zhang LB, Zhu Q, Wu ZQ, Ross-Ibarra J, Gaut BS, Ge S, et al. Selection on grain shattering genes and rates of rice domestication. New Phytol. 2009:184(3):708-20.

46. Chen LJ, Lee DS, Song ZP, Suh HS, Lu BR. Gene flow from cultivated rice (Oryza sativa) to its weedy and wild relatives. Ann Bot. 2004:93(1):67-73.

47. Liu X, Huang M, Fan B, Buckler ES, Zhang Z. Iterative usage of fixed and random effect models for powerful and efficient genome-wide association studies. PLoS Genet. 2016;12(2):e1005767.

48. Li Z, Rutger J. Geographic distribution and multilocus organization of isozyme variation of rice (Oryza sativa L.). Theor Appl Genet. 2000;101(3):379-87.

49. Team RC. R: A language and environment for statistical computing. Vienna: R Foundation for Statistical Computing; 2013. p. 2014. 
50. Lincoln S, Daly M, Lander E. Constructing genetics maps with MAPMAKER/EXP 3.0. Whitehead Technical Report. Cambridge, MA: MAPMAKER; 1992.

51. Li H, Ye G, Wang J. A modified algorithm for the improvement of composite interval mapping. Genetics. 2007;175(1):361-74.

52. Paterson AH, DeVerna JW, Lanini B, Tanksley SD. Fine mapping of quantitative trait loci using selected overlapping recombinant chromosomes, in an interspecies cross of tomato. Genetics. 1990;124(3):735-42.

53. Wang $Z$, Chen $C, X u Y$, Jiang $R$, Han $Y, X u Z$, et al. A practical vector for efficient knockdown of gene expression in rice (Oryza sativa L.). Plant Mol Biol Rep. 2004;22(4):409-17.

54. Hiei Y, Ohta S, Komari T, Kumashiro T. Efficient transformation of rice (Oryza sativa L.) mediated by Agrobacterium and sequence analysis of the boundaries of the T-DNA. Plant J. 1994;6(2):271-82.

55. Duan J, Zhang M, Zhang H, Xiong H, Liu P, Ali J, et al. OsMIOX, a myo-inositol oxygenase gene, improves drought tolerance through scavenging of reactive oxygen species in rice (Oryza sativa L.). Plant Sci. 2012;196:143-51.

56. Xiong H, Li J, Liu P, Duan J, Zhao Y, Guo X, et al. Overexpression of OsMYB48-1, a novel MYB-related transcription factor, enhances drought and salinity tolerance in rice. PLoS One. 2014;9(3), e92913.

57. Livak KJ, Schmittgen TD. Analysis of relative gene expression data using real-time quantitative $P C R$ and the $2-\Delta \Delta C T$ method. Methods 2001;25(4):402-8.

58. Thornton K. Libsequence: a C++ class library for evolutionary genetic analysis. Bioinformatics. 2003;19(17):2325-7.

59. Excoffier $L$, Lischer HE. Arlequin suite ver 3.5: a new series of programs to perform population genetics analyses under Linux and Windows. Mol Ecol Resour. 2010;10(3):564-7.

60. Teacher AG, Griffiths DJ. HapStar: automated haplotype network layout and visualization. Mol Ecol Resour. 2011;11(1):151-3.

61. Zhang H, Gao S, Lercher MJ, Hu S, Chen WH. EvolView, an online tool for visualizing, annotating and managing phylogenetic trees. Nucleic Acids Res. 2012:40(Web Server issue):W569.

\section{Submit your next manuscript to BioMed Central and we will help you at every step:}

- We accept pre-submission inquiries

- Our selector tool helps you to find the most relevant journal

- We provide round the clock customer support

- Convenient online submission

- Thorough peer review

- Inclusion in PubMed and all major indexing services

- Maximum visibility for your research

Submit your manuscript at www.biomedcentral.com/submit 